\title{
On the Abundance of Aperiodic Behaviour for Maps on the Interval
}

\author{
P. Collet ${ }^{1}$ and J.-P. Eckmann ${ }^{2}$
}

${ }^{1}$ Department of Physics, Harvard University, Cambridge, MA 02138 USA, and ${ }^{2}$ Département de Physique Théorique, Université de Genève, CH-1211 Genève 4, Switzerland

\section{Introduction}

In this paper, we study the following problem: Given a one-parameter family of continuous maps of the interval $[0,1]$ into itself, how many of these maps show aperiodic behaviour? For a particular family of maps containing a quadratic part we are able to show that for many values of the parameter (in fact for a set of positive Lebesgue measure) these maps do present aperiodic behaviour.

The parameter in question will be called $\delta$ (and is always supposed to be small, positive) and the particular family of functions is defined by

$$
f_{\delta}(x)= \begin{cases}2 x & \text { if } 0 \leqq x \leqq \frac{1}{2}-\delta \\ 2(1-x) & \text { if } \frac{1}{2}+\delta \leqq x \leqq 1 \\ 1-\delta-\left(x-\frac{1}{2}\right)^{2} / \delta & \text { if } x \in E_{\delta},\end{cases}
$$

where $E_{\delta}=\left\{x|| x-\frac{1}{2} \mid<\delta\right\}$, so the graph of $f_{\delta}$ is

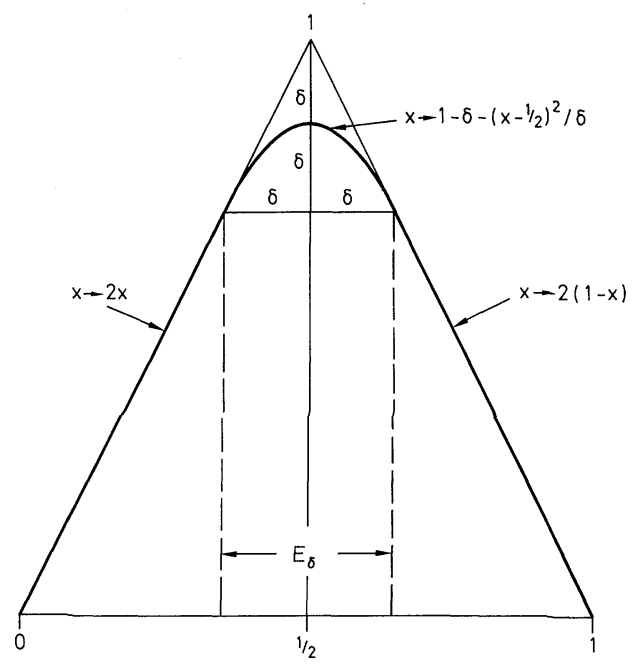

Fig. 1. The function $f_{\delta}$ for $\delta=0.15$ 
We shall analyze in great detail the successive iterates $x_{n}=f_{\delta}^{n}\left(\frac{1}{2}\right)$ of the point $\frac{1}{2}$, and in particular the derivative $D_{n, \delta}=\left.f_{\delta}^{n \prime}\right|_{x=f_{\delta}(1 / 2)}$. We shall show that for a large set of $\delta,\left|D_{n, \delta}\right|$ diverges exponentially, i.e. $\left|D_{n, \delta}\right|>2^{n / 7}$. This implies together with the fact that $f_{\delta}^{n}\left(\frac{1}{2}\right)$ does not return with a strict period to $E_{\delta}$ that $f_{\delta}$ has sensitive dependence with respect to initial conditions in the sense of Guckenheimer. Namely, this says that there is an $\varepsilon>0$ such that for every $x$ there is at least one (in fact very many) $y$ arbitrarily close to $x$ such that $\left|f_{\delta}^{n}(x)-f_{\delta}^{n}(y)\right|>\varepsilon$ for some $n>0$. We thus conclude that the "aperiodic" behaviour, observed numerically for many such maps, is a quite "common" thing. Such a result has been predicted, based on experimental evidence by several authors, cf. in particular Shaw [1] and Lorenz [2].

Fix a small $\delta>0$. We analyze the function $f=f_{\delta}$. Let $x=\frac{1}{2}+\mu_{i-1} \delta$, with $\left|\mu_{i-1}\right|$ $<1$. Define $n_{i}$ to be the smallest value of $n$ for which $\left|f^{n}(x)-\frac{1}{2}\right|<\delta$, and let $\delta \mu_{i}$ $=f^{n_{i}}(x)-\frac{1}{2}$. We shall establish a relation between $\mu_{i}, \mu_{i-1}$ and $n_{i}$. From the definition of $f$ we have

$$
\begin{aligned}
f(x) & =1-\delta\left(1+\mu_{i-1}^{2}\right) \\
f^{2}(x) & =2 \delta\left(1+\mu_{i-1}^{2}\right) .
\end{aligned}
$$

We claim that for $2 \leqq n \leqq n_{i}, f^{n}(x)$ can be written in the form

$$
f^{n}(x)=2 A+\sigma 2^{n-1}\left(1+\mu_{i-1}^{2}\right) \delta,
$$

with $\sigma= \pm 1$ and $A \in \mathbb{Z}$.

(Proof. The assertion is true for $n=2$. If it is true for $f^{n}$, then with $y=f^{n}(x)$,

$$
f^{n+1}(x)=\left\{\begin{array}{lll}
2(2 A)+\sigma 2^{n}\left(1+\mu_{i-1}^{2}\right) \delta, & \text { if } & 0 \leqq y \leqq \frac{1}{2}-\delta \\
2-4 A-\sigma 2^{n}\left(1+\mu_{i-1}^{2}\right) \delta, & \text { if } & \frac{1}{2}+\delta \leqq y \leqq 1,
\end{array}\right.
$$

so that the assertion follows for $n+1$.) Let

$$
\begin{aligned}
f^{n_{i}}(x) & =2 A_{i}+\sigma_{i} 2^{n_{i}-1}\left(1+\mu_{i-1}^{2}\right) \delta \\
& =\frac{1}{2}+\mu_{i} \delta .
\end{aligned}
$$

This also reads

$$
\delta\left[2^{n_{i}-1}\left(1+\mu_{i-1}^{2}\right)-\sigma_{i} \mu_{i}\right]=\sigma_{i}\left[\frac{1}{2}-2 A_{i}\right],
$$

or

$$
\delta=\frac{B_{i}}{2^{n_{i}-1}\left(1+v_{i-1}^{2}\right)+v_{i}},
$$

where $B_{i}=\sigma_{i}\left(\frac{1}{2}-2 A_{i}\right) \in \mathbb{N}-\frac{1}{2}$ (since $\delta>0$, and $\left|v_{i}\right|<1, B_{i}$ must be positive) and $v_{i}=$ $-\sigma_{i} \mu_{i}$. We are interested in $f^{n}\left(\frac{1}{2}\right)$, so that we set $\mu_{0}=v_{0}=0$, i.e.

$$
\delta=\frac{B_{1}}{2^{n_{1}-1}+v_{1}} .
$$

Note. Given $B_{i} \in \mathbb{N}-\frac{1}{2}, \sigma_{i}$ and $A_{i}$ are defined uniquely by

$$
\begin{aligned}
& A_{i}=n+1, \sigma_{i}=-1 \quad \text { if } \quad B_{i}=2 n+1+\frac{1}{2}, \\
& A_{i}=-n, \sigma_{i}=+1 \quad \text { if } B_{i}=2 n+\frac{1}{2} \text {. }
\end{aligned}
$$


These numbers (they are all functions of $\delta$ ), are related to the derivative by

$$
\left|\frac{d}{d x} f_{\delta}^{m}\right|_{x=f_{\delta}(1 / 2)}\left|=\prod_{j=1}^{k} 2^{n_{j}}\right| v_{j} \mid \cdot 2^{m^{\prime}},
$$

where $k$ is defined by

$$
\sum_{j=1}^{k} n_{j}<m<\sum_{j=1}^{k+1} n_{j}
$$

and $m^{\prime}=m-\sum_{j=1}^{k} n_{j}$. (The factor $\left|v_{k}\right|$ is absent if $m^{\prime}=0$.) Since we are interested in large derivatives, we must be especially careful when a $v_{j}$ is near 0 . In particular, if $v_{j}=0$ for $j>0$, we are in the presence of a stable periodic orbit for the value of $\delta$ in question, and we discard this value of $\delta$, together with a small interval around it. The problem is to choose these intervals sufficiently small so that their union has a relatively small volume, and sufficiently large so that $D_{n, \delta}$ diverges exponentially. The main content of this paper is that these two conditions are compatible. In the study of the excluded volume, we learn a lot about the "typical" behaviour of an aperiodic map, and this section is written in a self contained fashion so that the general ideas of the proof should be more easily grasped.

As an example of what is not a "typical" $\delta$, we show that there is an uncountable set of $\delta$ for which $f^{n}\left(\frac{1}{2}\right) \notin E_{\delta}$ for all $n>0$, [so that $\left|f^{n}\left(f\left(\frac{1}{2}\right)\right)\right|=2^{n}$ ], but that this set of $\delta$ has Lebesgue measure zero. Namely let $2^{-q-1} \leqq \delta<2^{-q}$ be such that the binary representation of $2^{q} \delta$ does not have $q-2$ consecutive zeros, nor $q-2$ consecutive " 1 "'s. Then

$$
f^{n}\left(\frac{1}{2}\right)-\frac{1}{2}=2 A_{n} \pm 2^{n-1} \delta-\frac{1}{2}
$$

and since $A_{n} \in \mathbb{Z}$, we have from the condition on $\delta$, that the fractional part of $f^{n}\left(\frac{1}{2}\right)$ $-\frac{1}{2}$ cannot have more than $q-1$ consecutive zeros, hence $\left|f^{n}\left(\frac{1}{2}\right)-\frac{1}{2}\right|>\delta$. On the other hand, the measure of the set of $\delta$ without $q-2$ consecutive zeros or 1's in their binary representation is zero since it is a subset of the numbers without "digit" $\underbrace{0 \ldots 0}_{q-2}$ and $\underbrace{1 \ldots 1}_{q-2}$ in their $2^{q-2}$-adic representation.

As a first step in describing the excluded volume, we standardize the description of orbits and introduce "resonances" (values of $p$ for which $v_{p}$ is very near to zero) and "blocked positions" (the returns to $E_{\delta}$ after $p$ in which the images of $\frac{1}{2}$ and of $x=\frac{1}{2}+\mu_{p} \delta$ have not yet separated).

\section{Definitions}

I. The set $\mathbb{P}$ of primitive resonances depends on $\delta$ through the numbers $n_{i}, B_{i}, v_{i}$. It is defined by

$$
\mathbb{P}=\left\{p \in \mathbb{N} \mid n_{p+1}=n_{1} \quad \text { and } \quad B_{p+1}=B_{1} \quad \text { and } \quad v_{p}^{2}<2^{-n_{1}} \Delta^{-4} L^{2}(p)\right\},
$$


where $\Delta=|\log \delta|$,

$$
L(n)=\prod_{j=n+1}^{\infty}\left(1+j^{-3}\right) .
$$

(We shall see in Lemma 1.4 below that the condition $B_{p+1}=B_{1}$ above is redundant.) The intuitive meaning of a resonance is that it is a return to $E_{\delta}$ which is a) very near to $v_{p}=0$ and b) sufficiently near to $v_{0}=0$ so that the trajectory to the next return takes the same number of steps $\left(n_{p+1}=n_{1}\right)$ and the same left-right sequence with respect to the maximum $\left(B_{p+1}=B_{1}\right)$. Note that $1<L(n)<3.3$.

II. When $p \in \mathbb{P}$, then $v_{p}$ is near $v_{0}=0$ and this means that we have almost encountered a stable periodic orbit. We shall now devise a test which finds the first $q>p$ for which the orbits starting from $v_{p}$ and from $v_{0}$ separate again (provided we exclude a "small" set of $\delta$ ). By definition, the test $\mathbf{T}(p, p)$ is true (passes). Then $\mathbf{T}(p, q)$ is recursively defined by

$$
\begin{aligned}
& \mathbf{T}(p, q) \text { is true if }\left[n_{q+1}=n_{q-p+1} \text { and } B_{q+1}=B_{q-p+1}\right. \\
& \text { and } \mathbf{T}(p, q-1) \text { is true and } \operatorname{sign} v_{q}=\operatorname{sign} v_{q-p} \\
& \text { and }[\text { if } q \in \mathbb{P} \text { then } \\
& \left.\left.\left|v_{q}\right|>\left|v_{q-p}\right|\left(1-\frac{1}{2}(q-p)^{-3}\right)\right]\right] .
\end{aligned}
$$

This test says first of all that the two orbits are considered to be blocked if they return simultaneously to $E_{\delta}$ and have the same left-right sequence. Furthermore if $q$ is a resonance, then $\left|v_{q}\right|$ must not be much smaller than $\left|v_{q-p}\right|$. We shall see in Lemma 4.1 below that, vaguely speaking, $v_{q} \sim v_{q-p}$ when $q$ is a blocked position.

III. We now define the resonance set $\mathbb{P}^{\prime}$.

1) Let $p_{0}$ be the smallest element of $\mathbb{P}$. Then $p_{0}$ is the smallest element of $\mathbb{P}^{\prime}$.

2) If $p \in \mathbb{P}^{\prime}$, define $t(p)$ as the smallest integer after $p$ for which $\mathbf{T}(p, t)$ does not hold, i.e.

$$
t(p)=\inf \{s \mid s>p \text { and } \mathbf{T}(p, s) \text { is not true }\} .
$$

Then the element of $\mathbb{P}^{\prime}$ following after $p$ is $f(p)$, where

$$
f(p)=\inf \{s \mid s \in \mathbb{P} \text { and } s \geqq t(p)\} .
$$

As an example:
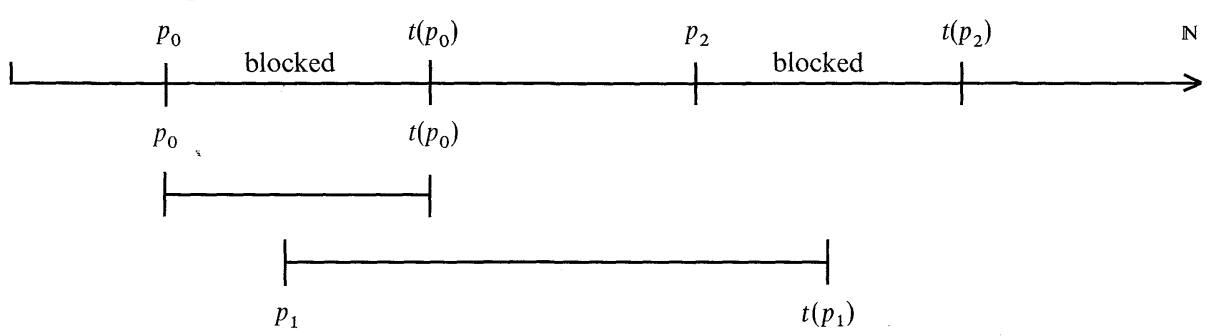

$p_{0}, p_{1}, p_{2} \in \mathbb{P}$,

$p_{0}, p_{2} \in \mathbb{P}^{\prime}, p_{2}=f\left(p_{0}\right)$.

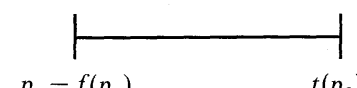


IV. The function $q$ counts the number of "unblocked" positions between $t(p)$ and $f(p)$. The formal definition is

$$
\begin{aligned}
& q(t)=t \quad \text { if } t<p_{0}, \\
& q(t)=q(t-1) \quad \text { if } \quad p \leqq t<t(p) \quad \text { for some } \quad p \in \mathbb{P}^{\prime} \text {, } \\
& q(t)=q(t-1)+1 \text { if } t(p) \leqq t<f(p) \text { for some } p \in \mathbb{P}^{\prime} \text {. }
\end{aligned}
$$

The function $r$ counts the number of $p \in \mathbb{P}$

$$
r(s)=\operatorname{card}\{p \mid p \in \mathbb{P}, p<s\}+1 .
$$

V. We next describe five situations in which we exclude a set of $\delta$. Recall that all the quantities $B_{i}, n_{i}, \ldots$ are functions of $\delta$ and that $\Delta=|\log \delta|$.

For every $j \in \mathbb{N}$, we define

$$
\begin{aligned}
\mathbf{I}_{j}^{1}= & \left\{\delta \mid 0 \leqq \delta \leqq \delta_{0} \text { and }\left|v_{j}\right| \leqq \Delta^{-4 j}\right. \text { and } \\
& \left.\left(j=1 \text { or } j \leqq p_{0} \text { or } t(p) \leqq j \leqq f(p) \text { for some } p \in \mathbb{P}^{\prime}\right)\right\}, j=1,2, \ldots .
\end{aligned}
$$

Of course, one of the problems will be to describe this exclusion as a function of $\delta$. The idea of excluding $\mathbf{I}_{j}^{1}$ is to avoid for the unblocked positions and for $j \in \mathbb{P}^{\prime}$ and for $j=t(p)$, when $p \in \mathbb{P}^{\prime}$ that $\left|v_{j}\right|$ gets too small. The larger the number of returns already encountered, the smaller we choose the excluded volume. No exclusion is necessary when $j$ is blocked, because we shall derive from $v_{j} \sim v_{j-p}$ [where $p<j<t(p)]$ that $\left|v_{j}\right|$ does not become too small, since $\left|v_{j-p}\right|$ does not.

For every $j \in \mathbb{N}$, we define

$$
\begin{aligned}
\mathbf{I}_{j}^{2}= & \left\{\delta \mid 0 \leqq \delta \leqq \delta_{0} \text { and } 1-\left|v_{j}\right| \leqq \Delta^{-15 j}\right. \\
& (\text { if } j=1 \text { replace }-15 \text { by }-3)\}, j=1,2, \ldots .
\end{aligned}
$$

This exclusion has the purpose of avoiding $v_{j}$ (for all $j$ ) to be too near to \pm 1 . The problem with which this is connected is the following. We would like to argue that when $n_{q+1} \neq n_{q-p+1}$ then $v_{q}-v_{q-p}$ is not too small. What may happen, however, is that after $n_{q-p+1}$ steps the image of $\frac{1}{2}+\delta \mu_{0}=\frac{1}{2}$ is near to the boundary of $E_{\delta}$ while $f^{n_{q-p+1}}\left(\frac{1}{2}+\delta \mu_{p}\right)$ just very nearly "misses" $E_{\delta}$. In fact, the exclusion $\mathbf{I}_{j}^{2}$ handles the case $n_{q+1}>n_{q-p+1}$ while the exclusion $\mathbf{I}_{j}^{3}$ below deals with the case when $n_{q+1}$ $<n_{q-p+1}$ and prevents $f^{n_{q+1}}\left(\frac{1}{2}+\delta \mu_{0}\right) \notin E_{\delta}$ from approaching $E_{\delta}$ too much from the outside.

$$
\mathbf{I}_{j}^{3}=\left\{\delta \mid 0 \leqq \delta \leqq \delta_{0} \text { and } \exists n<n_{j+1}, \exists B\right.
$$

compatible with $n, \delta, \exists \varepsilon \in\{1,-1\}$, such that

$$
\left.\left|\delta\left(1+v_{j}^{2}+\varepsilon 2^{-n+1}\right)-B 2^{-n+1}\right| \leqq 4 \delta 2^{-n} \Delta^{-15 j-3}\right\}, j=0,1,2, \ldots
$$

Here, a number $B$ is called compatible with $n, \delta$, if there is an orbit of $f_{\delta}$ for which the pair $B, n$ actually occurs in a first return. The precise definition is:

A number $B \in \mathbb{N}-\frac{1}{2}$ is compatible with $n, \delta$ if there is an $x, 1 \leqq x<2$, such that

1) $\left|x \delta-B 2^{-n+1}\right|<2^{-n+1} \delta$,

2) For all $n^{\prime}<n$ and all $B^{\prime} \in \mathbb{N}-\frac{1}{2}$,

$\left|x \delta-B^{\prime} 2^{-n^{\prime}+1}\right| \geqq 2^{-n^{\prime}+1} \delta$. 

We shall now define a further set of $\delta$ which will be excluded. Let $\sum_{b, s}$ be the sum
over the blocked indices $\leqq s$, i.e. over

$\left\{j \mid j \leqq s\right.$ and for some $\left.p \in \mathbb{P}^{\prime}, p \leqq j \leqq t(p)-1\right\}$.

Then we define

$$
\begin{aligned}
\mathbf{I}_{s}^{4}= & \left\{\delta \mid 0 \leqq \delta \leqq \delta_{0}\right. \text { and } \\
& \left.\sum_{j} b, s n_{j+1}>10000 q(s) \log _{2} \Delta\right\}, \quad s=1,2, \ldots .
\end{aligned}
$$

This set excludes in essence those $\delta$ for which a non zero fraction of positions is blocked or for which long returns are blocked. We shall see on p. 123, that on the complement of $\mathbf{I}_{s}^{4}$, one has

$$
s<q(s)(1+\varepsilon)
$$

for some small $\varepsilon>0$. Therefore a "typical" orbit is unblocked most of the time. We shall also derive, in Lemma 4.4, the inequality

$$
t(p)-p \leqq p,
$$

which says that if a resonance occurs after $p$ returns, a "typical" orbit is not blocked for more than $p$ returns. On the other hand, it seems to us that if we single out those $\delta$ for which only a finite number of blocked positions occurs, or for which only blocked sequences of bounded length occur, then the Lebesgue measure of these $\delta$ is zero.

Our last exclusion is the set $\mathbf{I}_{s}^{5}$ (which has in fact zero Lebesgue measure among those $\delta$ remaining after the other exclusions). It is essentially

$$
\begin{gathered}
\mathbf{I}_{s}^{5}=\left\{\delta \mid 0 \leqq \delta \leqq \delta_{0} \text { and } s \mathbb{N} \subset\left\{m_{i}\right\}_{i=1,2, \ldots},\right. \text { where } \\
\left.m_{i}=\sum_{k=1}^{i} n_{i}\right\}, s=1,2, \ldots,
\end{gathered}
$$

see Sect. 7 for a precise definition. This excludes those $\delta$ for which $f^{k s}\left(\frac{1}{2}\right) \in E_{\delta}$ for $k=1,2, \ldots$, i.e. for which the image of the maximum periodically returns to $E_{\delta}$.

\section{Results}

Define now $\mathbf{J}\left(\delta_{0}\right)=\left\{\delta \mid 0 \leqq \delta \leqq \delta_{0}\right.$ and $\left.\delta \notin \mathbf{I}_{s}^{l}, \mathrm{~s}=0,1,2, \ldots, l=1,2,3,4,5\right\}$. Our results are

Theorem A. For $\delta_{0}>0$ sufficiently small, the Lebesgue measure of $\mathbf{J}\left(\delta_{0}\right)$ is at least $\delta_{0}\left(1-1 / \log \left(\delta_{0}^{-1}\right)\right)$.

Theorem B. For $\delta_{0}>0$ sufficiently small and for all $\delta \in \mathbf{J}\left(\delta_{0}\right)$ one has

(1) $\left|f_{\delta}^{n \prime}\right|_{x=f_{\delta}(1 / 2)} \mid>2^{n / 7}$.

(2) $f_{\delta}$ is topologically conjugate to a piecewise linear map $g_{\tau}: x \rightarrow \tau\left(\frac{1}{2}-\left|x-\frac{1}{2}\right|\right)$, with $\tau>\sqrt{2}$. 
(3) $f_{\delta}$ is sensitive to initial conditions: i.e. for every $x \in[0,1]$ and small $\varepsilon>0$ there is in every neighborhood $U$ of $x$ a $y$ and a number $n$ such that $\left|f_{\delta}^{n}(x)-f_{\delta}^{n}(y)\right|>\varepsilon$.

The proofs of these facts take up the remainder of our paper and we outline here the main steps.

In Sect. 1, we warm up with some easy observations, among which the most farreaching is that some minimum time is required between two returns to $E_{\delta}$; namely

$$
2^{n_{j}}>\delta^{-1} / 8
$$

In Sect. 2, we prepare the tools for the comparison of $v_{q}$ with $v_{q-p}$ when $q$ is blocked. The main observations are:

If $q \notin \mathbb{P}$ then

$$
\left|v_{q}\right| \geqq 2^{-n_{q+1} / 2} \Delta^{-2},
$$

(i.e. non-resonant $v$ 's are not arbitrary small). Also, as long as $q$ is blocked, due to the basic identity $(0.1)$,

$$
v_{p}^{2}=\prod_{j=p+1}^{q-1} \frac{1}{2^{n_{j}-1}\left(\left|v_{j}\right|+\left|v_{j-p}\right|\right)} \cdot \frac{\left|v_{q}-v_{q-p}\right|}{2^{n_{q-1}}},
$$

(i.e. $v_{p}$, which is resonant, can be estimated through the square root of $\left|v_{q}-v_{q-p}\right|$ ).

In Sect. 3, we bound the quotient

$$
\prod_{j=p+1}^{q-1}\left|v_{j} / v_{j-p}\right| \sim \Delta^{-4},
$$

and we give bounds on $\left|v_{q}-v_{q-p}\right|$, using the exclusions. This serves in Sect. 4 to bound recursively the derivative as follows. From the calculations on p. 117 we see that a quantity of interest is

$$
R_{m}=\prod_{j=1}^{m}\left|v_{j}\right| 2^{n_{j+1}} .
$$

Assume we have already bounded $R_{p-1}$. Then the main result is that, essentially due to $(0.8)$,

$$
R_{t(p)-1} \sim \Delta^{-8} R_{p-1}\left(2^{n_{1}} R_{t(p)-p-1}\right)^{1 / 2}\left|v_{t(p)}-v_{t(p)-p}\right|^{1 / 2}
$$

i.e. we have absorbed the potentially dangerous factor $v_{p}$ into the last two factors. Using now the bounds on $\left|v_{q}-v_{q-p}\right|$ established in Sect. 3, we get in Sect. 5 the exponential divergence of $R_{n}$, i.e. Theorem $\mathrm{B} 1$.

In Sect. 6 we bound the excluded volumes. The idea is to measure how fast a resonant interval is traversed when $\delta$ varies (very little). The relevant bound shows that

$$
\left|\frac{d v_{s}}{d \delta}\right|>\frac{2^{n_{1}} R_{s-1}}{4 \delta}
$$

and then we use the formula

$$
\int d \delta \sim \int d v_{s}\left|\frac{d \delta}{d v_{s}}\right| \operatorname{card}\left[v_{s}^{-1}\left(v_{s}\right)\right]
$$


to bound the integrals. We need a relatively fine decomposition of $0<\delta<\delta_{0}$ in order to get the desired bound of Theorem A.

In Sect. 7, we use the exclusion of $\mathbf{I}_{s}^{5}$ to reduce the discussion to the analysis done by Guckenheimer [3], and this yields Theorem B2 and B3. In particular there is a homeomorphism $h$ (which is not necessarily absolutely continuous) such that $h \circ f_{\delta}=g_{\tau} \circ h$.

It seems to us that most of the preceding results carry over without much change to similar families of functions which are suitable small perturbations of the special family we have chosen. Note that our particular choice has been motivated through the following consideration: the often studied maps $x \mapsto 4 s x(1-x)$, with $s=1-\pi^{2} \delta^{2} / 4$ are conjugated [through $x=\sin ^{2}(\pi y / 2)$ ] to $y \mapsto(2 / \pi) \arcsin \left(s^{1 / 2} \sin (\pi y)\right) \equiv \hat{f}_{\delta}(y)$. Then $\hat{f}_{\delta}$ is similar to $f_{\delta}$ in that for small $y, \hat{f}_{\delta}\left(\frac{1}{2}+y\right)=1-\delta-\frac{2}{\delta} y^{2}+\mathcal{O}\left(\delta^{2}\right)$.

Note. Similar results have been announced by Jakobson.

\section{Preliminary Remarks}

Lemma 1.1. We have

$$
2^{n_{j}} \geqq 1 /(8 \delta) \text {. }
$$

Proof. By definition, with $j>1$, and $N_{k}=\sum_{l=1}^{k} n_{l}$,

$$
\left|f_{\delta}^{N_{j-1}}\left(\frac{1}{2}\right)-\frac{1}{2}\right|<\delta,
$$

so that

$$
f_{\delta}^{N_{j-1}+1}\left(\frac{1}{2}\right) \geqq 1-2 \delta
$$

and

$$
f_{\delta}^{N_{j-1}+k+1}\left(\frac{1}{2}\right) \leqq 2^{k+1} \delta, \quad k>0,
$$

as long as $2^{k} \delta<\frac{1}{2}-\delta$. For $\delta_{0}$ sufficiently small, this implies

$$
2^{n_{j}+1} \delta \geqq \frac{1}{4} \text {. }
$$

Lemma 1.2. We have

$$
\delta / 4 \leqq B_{1} / 2^{n_{1}} \leqq \delta
$$

Proof. From

$$
\delta=B_{1} /\left(2^{n_{1}-1}+v_{1}\right),
$$

we deduce

$$
\delta\left(1+v_{1} / 2^{n_{1}-1}\right)=B_{1} / 2^{n_{1}-1} .
$$

The assertion follows from Lemma 1.1. 
Lemma 1.3. If $j>1$, then with

$$
Q=B_{j} 2^{n_{1}} /\left(B_{1} 2^{n_{j}}\right),
$$

one has $\frac{1}{3} \leqq Q \leqq 3$.

Proof. By (0.1), (0.2),

$$
Q=\frac{1+v_{j-1}^{2}+v_{j} 2^{1-n_{j}}}{1+v_{1} 2^{1-n_{1}}} .
$$

The assertion follows, if $\delta_{0}$ is sufficiently small, from Lemma 1.1.

We next present two simple consequences of the absence of a resonance condition.

Lemma 1.4. If $n_{s+1}=n_{t+1}$ and $B_{s+1} \neq B_{t+1}$, for some $s, t \geqq 0$, then

$$
\left|v_{s}^{2}-v_{t}^{2}\right| \geqq 2^{-n_{s+1}-1} / \delta \text {. }
$$

(Here, $v_{0}=0$.)

Proof. By Eq. (0.1), we have with $n=n_{s+1}=n_{t+1}$,

$$
v_{s}^{2}-v_{t}^{2}=\left(B_{s+1}-B_{t+1}\right) /\left(\delta 2^{n}\right)-\left(v_{s+1}-v_{t+1}\right) / 2^{n-1} .
$$

By Lemma 1.1, we find

$$
\left|v_{s}^{2}-v_{t}^{2}\right| \geqq 2^{-n}\left(\delta^{-1}-32\right) \geqq 2^{-n-1} / \delta .
$$

Lemma 1.5. If $n_{s+1}>n_{t+1}$, then

$$
\begin{aligned}
& \quad\left|v_{t+1}+2^{n_{t+1}-1}\left(v_{t}^{2}-v_{s}^{2}\right)\right| \geqq 1 . \\
& \text { (Here, } \left.v_{0}=0 .\right)
\end{aligned}
$$

Proof. From Eq. (0.1), we have

$$
\delta v_{t+1}=B_{t+1}-2^{n_{t+1}-1}\left(1+v_{t}^{2}\right) \delta,
$$

i.e.,

$$
\left(v_{t+1}+2^{n_{t+1}-1}\left(v_{t}^{2}-v_{s}^{2}\right)\right) \delta=B_{t+1}-2^{n_{t+1}-1}\left(1+v_{s}^{2}\right) \delta .
$$

Since $B_{s+1}$ is compatible with $n_{s+1}, \delta$, we have for all $n<n_{s+1}$ with $x=1+v_{s}^{2}$,

$$
\left|2^{n-1} x \delta-B_{t+1}\right| \geqq \delta
$$

but this implies the assertion of the lemma.

Lemma 1.6. For $\delta \notin \mathbf{I}_{s}^{4}$ and $\varepsilon>0$ one has for $\delta_{0}<\delta_{0}(\varepsilon)$,

$$
q(s) \geqq s(1-\varepsilon) \geqq r(s)(1-\varepsilon) .
$$

Proof. From $\delta \notin \mathbf{I}_{s}^{4}$ we have

$$
\sum_{j} b, s n_{j+1} \leqq 10000 q(s) \log _{2} \Delta \text {. }
$$

But, by Lemma $1.1, n_{j+1}>\Delta / 2$. Therefore

$$
\sum_{j} b, s n_{j+1}>(\Delta / 2)(s-q(s)) \text {. }
$$


Thus

$\Delta s / 2<q(s)\left(\Delta / 2+10000 \log _{2} \Delta\right)$,

and the assertion follows for sufficiently small $\delta_{0}$.

\section{First Consequences}

In this section, we collect several estimates which are relatively straightforward consequences of the definitions; in particular, we defer the recursive bounds to a later section.

We define

$$
\begin{aligned}
\mathbf{J}_{s}^{4}= & \left\{\delta \mid 0 \leqq \delta \leqq \delta_{0} \quad\right. \text { and } \\
& \left.\delta \notin \mathbf{I}_{j}^{k}, j=0, \ldots, s ; k=1, \ldots, 4\right\} .
\end{aligned}
$$

Lemma $2.1^{1}$. Let $\delta>0$.

(1) If $p \in \mathbb{P}^{\prime}$ then

$$
v_{p}^{2}=\left(v_{1}-v_{p+1}\right) / 2^{n_{p+1}-1} .
$$

(2) If $p \in \mathbb{P}^{\prime}$ and $p<s<t(p)$ then

$$
\left|v_{s}-v_{s-p}\right|=\left|v_{s+1}-v_{s-p+1}\right| /\left(2^{n_{s+1}-1}\left(\left|v_{s}\right|+\left|v_{s-p}\right|\right)\right) \text {. }
$$

(3) If $p \in \mathbb{P}^{\prime}$ and $p<s \leqq t(p)$ then

$$
v_{p}^{2}=\frac{\left|v_{s}-v_{s-p}\right|}{2^{n_{s}-1}} \prod_{j=p+1}^{s-1}\left(2^{n_{j}-1}\left(\left|v_{j}\right|+\left|v_{j-p}\right|\right)\right)^{-1} .
$$

Proof. (1) is an immediate consequence of $n_{p+1}=n_{1}$ and $B_{p+1}=B_{1}$ and of (0.1). To prove (2), we note that $p<s<t(p)$ implies $n_{s+1}=n_{s-p+1}$ and $B_{s+1}=B_{s-p+1}$. Therefore (0.1) implies

$$
\begin{aligned}
v_{s}-v_{s-p} & =\left(v_{s}^{2}-v_{s-p}^{2}\right) /\left(v_{s}+v_{s-p}\right) \\
& =-\left(v_{s+1}-v_{s-p+1}\right) 2^{1-n_{s+1}} /\left(v_{s}+v_{s-p}\right),
\end{aligned}
$$

and the assertion (2) follows now because $p<s<t(p)$ implies $v_{s} v_{s-p}>0$. (3) follows now by induction.

Lemma 2.2. Given $\delta>0$, let $p \in \mathbb{P}^{\prime}$ and $p<p+j<t(p)$ and $j \in \mathbb{P}$. Then either

$$
\left|v_{p+j}\right| \geqq\left|v_{j}\right|\left(1+\frac{1}{2} j^{-2}\right),
$$

or

$$
\left|v_{p+j}-v_{j}\right| \leqq \frac{1}{2} j^{-2}\left|v_{j}\right| .
$$

Proof. Suppose the contrary of (2.1). If $p+j \in \mathbb{P}$, then due to the condition $\mathbf{T}(p, p+j),\left|v_{p+j}\right|>\left|v_{j}\right|\left(1-\frac{1}{2} j^{-2}\right)$.

This and the contrary of (2.1) imply (2.2) since $v_{p+j} v_{j}>0$. If $p+j \notin \mathbb{P}$, then since $n_{p+j+1}=n_{j+1}=n_{1}$ and $B_{p+j+1}=B_{j+1}=B_{1}$, we must have

$$
\left|v_{p+j}\right|>2^{-n_{1} / 2} \Delta^{-2} L(p+j) \text {. }
$$

1 Empty products are defined to be equal to 1 
Since $L(j) \geqq 1$ for all $j \geqq 0$ we find

$$
\frac{\left|v_{p+j}\right|}{\left|v_{j}\right|}>\frac{L(p+j)}{L(j)} \geqq\left(\prod_{k=j+1}^{\infty}\left(1+k^{-3}\right)\right)^{-1} \text {. }
$$

But $\prod_{k=j+1}^{\infty}\left(1+k^{-3}\right) \leqq \exp \left(j^{-2} / 2\right)$, so that

$$
\left|v_{p+j}\right|>\left|v_{j}\right|\left(1-\frac{1}{2} j^{-2}\right) \text {. }
$$

Therefore one of the two alternatives must hold in this case, too.

Lemma 2.3. If $\delta \in \mathbf{J}_{q}^{4}$ and $q \notin \mathbb{P}$, then

$$
v_{q}^{2} \geqq 2^{-n_{q+1}} \Delta^{-4},
$$

where $\Delta=|\log \delta|$.

Proof. We distinguish four cases.

Case 1. $n_{q+1}=n_{1}$ and $B_{q+1}=B_{1}$. By the definition of $\mathbb{P}$, since $q \notin \mathbb{P}$, we have

$$
v_{q}^{2}>2^{-n_{1}} \Delta^{-4},
$$

and the assertion is evident.

Case 2. $n_{q+1}=n_{1}$ and $B_{q+1} \neq B_{1}$. Then the assertion follows from Lemma 1.4, and from $v_{0}=0$.

Case 3. $n_{q+1}<n_{1}$. By Lemma 1.5, we have

$$
\left|v_{q+1}+2^{n_{q+1}-1} v_{q}^{2}\right| \geqq 1 \text {. }
$$

Assume now $v_{q}^{2}<2^{-n_{q+1}+1} \Delta^{-3}$. Then we have for $\varepsilon=1$ or for $\varepsilon=-1$,

$$
\left|v_{q+1}+\varepsilon\right| \leqq \Delta^{-3} \text {. }
$$

But this implies, by $(0.1)$,

$$
\delta=B_{q+1} /\left(2^{n_{q+1}-1}+\varepsilon+A\right),
$$

with $|A| \leqq 2 \Delta^{-3}$, so that

$$
\left|\delta-B_{q+1} /\left(2^{n_{q+1}}+\varepsilon\right)\right| \leqq 2 \Delta^{-3} \delta /\left(2^{n_{q+1}}-1\right),
$$

and since $2^{n_{q+1}} \geqq \mathcal{O}\left(\delta^{-1}\right)$, by Lemma 1.1 , this is in contradiction with $\delta \in \mathbf{J}_{q}^{4}$ (since $\left.\delta \notin \mathbf{I}_{0}^{3}\right)$. This implies (1) in this case.

Case 4. $n_{q+1}>n_{1}$. Applying again Lemma 1.5, we have

$$
\left|v_{1}-2^{n_{1}-1} v_{q}^{2}\right| \geqq 1 \text {. }
$$

Assume now $v_{q}^{2}<2^{-n_{q+1}+1} \Delta^{-3}$. Then we have, as before

$$
\left|v_{1}+\varepsilon\right| \leqq \Delta^{-3}
$$

and this is in contradiction with $\delta \in \mathbf{J}_{q}^{4}$ (since $\delta \notin \mathbf{I}_{1}^{2}$ ). Hence the assertion follows in this case. 
Lemma 2.4. Let $\delta \in \mathbf{J}_{q}^{4}$, let $p \in \mathbb{P}^{\prime}$ and $q=p+j<t(p)$. Then

$$
\left|v_{p+j} / v_{j}\right| \geqq \Delta^{-3} \text {. }
$$

Proof. By Lemma 2.1 (2), we have

$$
\frac{\left|v_{p+j}-v_{j}\right|}{\left|v_{j}\right|}=\frac{\left|v_{p+j+1}-v_{j+1}\right|}{2^{n_{j+1}-1}\left|v_{j}\right|\left(\left|v_{j+p}\right|+\left|v_{j}\right|\right)} \leqq 4 \cdot 2^{-n_{j+1}}\left|v_{j}\right|^{-2} .
$$

If $p+j \notin \mathbb{P}$ and if we assume

$$
\frac{\left|v_{p+j}\right|}{\left|v_{j}\right|}<\Delta^{-3}
$$

then (since $n_{p+j+1}=n_{j+1}$ ), we have

$$
2^{n_{j+1}}\left|v_{j}\right|^{2}>2^{n_{j+1}}\left|v_{p+j}\right|^{2} \Delta^{6} \geqq \Delta^{2},
$$

by Lemma 2.3 , since $p+j \notin \mathbb{P}$ and $\delta \in \mathbf{J}_{p+j}^{4}$. Hence it follows

$$
\frac{\left|v_{p+j}\right|}{\left|v_{j}\right|} \geqq 1-\frac{\left|v_{p+j}-v_{j}\right|}{\left|v_{j}\right|}>1-4 \Delta^{-2}>\Delta^{-3},
$$

which is in contradiction with (2.3). If $p+j \in \mathbb{P}$, since $p+j \neq t(p)$, the test implies

$$
\left|v_{p+j} / v_{j}\right|>\left(1-\frac{1}{2} j^{-3}\right)>\Delta^{-3} \text {. }
$$

Lemma 2.5. If $\delta \in \mathbf{J}_{s}^{4}$, then for $s>0$,

$$
\left|v_{s}\right| \geqq \Delta^{-4 s} \text {. }
$$

Proof. By induction on $s$. The lemma is obvious for $s=1$ by $\delta \notin \mathbf{I}_{1}^{1}$. Assume the bound for all $s^{\prime}<s$. If $s$ is such that for some $p \in \mathbb{P}^{\prime}$ we have $t(p) \leqq s \leqq f(p)$ (or $\left.s \leqq p_{0}\right)$, then the bound follows from $\delta \notin \mathbf{I}_{s}^{1}$. In the opposite case, we use Lemma 2.4 and obtain

$$
\left|v_{s}\right| \geqq\left|v_{s} / v_{s-p}\right|\left|v_{s-p}\right| \geqq \Delta^{-3 \cdot} \Delta^{-4(s-p)},
$$

and the bound follows.

We now start the estimates which show that at the point $t(p)$, the values $v_{t(p)}$ and $v_{t(p)-p}$ are "well separated". This is of course due to the exclusions we have performed. Note however, that our procedure of "waiting" with the exclusion up to $t(p)$ instead of excluding the resonance condition at $p$ altogether is crucial in obtaining a sufficiently good bound on the volume of the excluded $\delta$ 's.

Lemma 2.6. Given $p$ and $s>p$ let $\delta \in \mathbf{J}_{s-p+1}^{4}$ be such that $p \in \mathbb{P}^{\prime}$ and $s=t(p)$.

(1) If $v_{s} v_{s-p}<0$ then

$$
\left|v_{s}-v_{s-p}\right|>\Delta^{-4(s-p)} \text {. }
$$

(2) If $n_{s+1}=n_{s-p+1}$ and $B_{s+1} \neq B_{s-p+1}$ and $v_{s} v_{s-p}>0$ then

$$
\left|v_{s}^{2}-v_{s-p}^{2}\right| \geqq 2^{-n_{s+1}-1} / \delta \text {. }
$$

(3) If $n_{s+1}>n_{s-p+1}$ and $v_{s} v_{s-p}>0$ then

$$
\left|v_{s}^{2}-v_{s-p}^{2}\right| \geqq 2^{-n_{s-p+1}} \Delta^{-15(s-p+1)} \text {. }
$$


(4) If $n_{s+1}<n_{s-p+1}$ and $v_{s} v_{s-p}>0$ then

$\left|v_{s}^{2}-v_{s-p}^{2}\right| \geqq 2^{-n_{s+1}} \Delta^{-15(s-p)-3}$.

(5) If $n_{s+1}=n_{s-p+1}$ and $B_{s+1}=B_{s-p+1}$ and $v_{s} v_{s-p}>0$ then $s=f(p)$ and $\left|v_{s}-v_{s-p}\right| \geqq \Delta^{-5(s-p)}$.

Proof. We distinguish five causes for which the test $\mathbf{T}(p, s)$ can fail.

Case 1. $v_{s} v_{s-p}<0$. Then

$$
\left|v_{s}-v_{s-p}\right|=\left|v_{s}\right|+\left|v_{s-p}\right| \geqq\left|v_{s-p}\right|,
$$

and the assertion follows from Lemma 2.5.

Case 2. $v_{s-p} v_{s-0}$ and $n_{s+1}=n_{s-p+1}$ and $B_{s+1} \neq B_{s-p+1}$. By Lemma 1.4, we have $\left|v_{s}^{2}-v_{s-p}^{2}\right| \geqq 2^{-n_{s+1}-1} \delta^{-1}$.

Case 3. $v_{s} v_{s-p}>0$ and $n_{s+1}>n_{s-p+1}$. By Lemma 1.5, we have

$$
\left|v_{s-p+1}+2^{n_{s-p+1}-1}\left(v_{s-p}^{2}-v_{s}^{2}\right)\right| \geqq 1 \text {. }
$$

Assume the contrary of the conclusion of (3). Then we must have

$$
1-\left|v_{s-p+1}\right|<\Delta^{-15(s-p-1)},
$$

and this contradicts $\delta \notin \mathbf{I}_{s-p+1}^{2}$.

Case 4. $v_{s} v_{s-p}>0$ and $n_{s+1}<n_{s-p+1}$. From Lemma 1.5, we have

$$
\left|v_{s+1}+2^{n_{s+1}-1}\left(v_{s}^{2}-v_{s-p}^{2}\right)\right| \geqq 1 \text {. }
$$

Assume again the contrary of the conclusion of (4). We find again

$$
1-\left|v_{s+1}\right| \leqq \Delta^{-15(s-p)-3} \text {. }
$$

From the Eq. (0.1), we derive, with $\varepsilon=+1$ or -1

$$
\begin{aligned}
\delta\left(1+v_{s-p}^{2}+\frac{\varepsilon}{2^{n_{s+1}-1}}\right)= & \frac{B_{s+1}}{2^{n_{s+1}-1}}-\delta\left(v_{s}^{2}-v_{s-p}^{2}\right) \\
& -\delta \frac{\left(v_{s+1}-\varepsilon\right)}{2^{n_{s+1}-1}}=\frac{B_{s+1}}{2^{n_{s+1}-1}}+A,
\end{aligned}
$$

and from (2.4), we find $|A| \leqq 4 \delta 2^{-n_{s+1}} \Delta^{-15(s-p)-3}$, and this contradicts $\delta \in \mathbf{J}_{s-p}^{4}$ (i.e. $\left.\delta \notin \mathbf{I}_{s-p}^{3}\right)$.

Case 5. $v_{s} v_{s-p}>0$ and $n_{s+1}=n_{s-p+1}$ and $B_{s+1}=B_{s-p+1}$. Since $s=t(p)$ the test $\mathbf{T}(p, s)$ must have failed. The only remaining possibility for this to occur is that $s \in \mathbb{P}$ (actually in $\mathbb{P}^{\prime}$ ) and

$$
\left|v_{s}\right| \leqq\left|v_{s-p}\right|\left(1-\frac{1}{2}(s-p)^{-3}\right) \text {. }
$$

Therefore $\left|v_{s}-v_{s-p}\right| \geqq \frac{1}{2}\left|v_{s-p}\right|(s-p)^{-3}$, and the assertion follows from Lemma 2.5. This completes the proof of the lemma. 
Corollary 2.7. Given $p$ and $s>p$, let $\delta \in \mathbf{J}_{s-p+1}^{4}$ be such that $p \in \mathbb{P}^{\prime}$ and $s=t(p)$. Then $\left|v_{s}-v_{s-p}\right| \geqq 2^{-n_{s+1}} \Delta^{-15(s-p+1)}$.

This is just the worst possible combined bound of Lemma 2.6.

Note. A direct calculation, using Eq. (0.1), and $\delta \notin \bigcup_{s=0}^{j} \mathbf{I}_{s}^{3}$ shows that

$$
\left|f^{m}\left(\frac{1}{2}\right)-\frac{1}{2}\right|-\delta>\delta \Delta^{-15(j+1)}
$$

for $m$ between return $j$ and return $j+1$.

\section{Comparison of Blocked Sequences}

In this section, we bound the quotient

$$
\prod_{j=1}^{s-p-1}\left|v_{p+j} / v_{j}\right|
$$

from below, where $p \in \mathbb{P}^{\prime}$ and $s=t(p)$. This bound will express the fact that as long as the orbits of $x_{p}=f^{N_{p}^{\prime \prime}}\left(\frac{1}{2}\right)$, where $N_{p}^{\prime \prime}=\sum_{j=1}^{p} n_{j}$, and of $\frac{1}{2}$ are very near to each other, the derivatives $\frac{d}{d x}\left(f^{N}\right)$, where $N+1=\sum_{j=p+1}^{s-p} n_{j}$, taken at $x=f\left(x_{p}\right)$ and at $x=f\left(\frac{1}{2}\right)$ should be about equal. In fact, due to the conditions $\mathbf{T}(.,$.$) , and by the chain rule,$ we find

$$
\left.\frac{d}{d x}\left(f^{N}\right)\right|_{x=f\left(x_{p}\right)}= \pm 2^{n_{p+1}} \prod_{j=1}^{s-p-1} v_{p+j} 2^{n_{p+j+1}},
$$

while

$$
\left.\frac{d}{d x}\left(f^{N}\right)\right|_{x=f(1 / 2)}= \pm 2^{n_{1}} \prod_{j=1}^{s-p-1} v_{j} 2^{n_{j+1}} .
$$

Now our interest in $\left|v_{p+j} / v_{j}\right|$ should become obvious from $n_{j+1}=n_{j+p+1}(j=0$, $1, \ldots, s-p-1)$. We next prepare the necessary tools to perform bounds when $s$ satisfies $p \leqq s \leqq t(p)$ for some $p \in \mathbb{P}^{\prime}$.

Define $t_{0}=p$ and denote by $t_{1}, \ldots, t_{m}$ the elements of $\{n \in \mathbb{N} \mid n-p \in \mathbb{P}$ and $p<n$ $\leqq s\}$ in ascending order. In the next two lemmas we only consider values of $\delta$ which produce this set.

Lemma 3.1. Let $\delta \in \mathbf{J}_{t_{k}-p}^{4}$. Let $l$ satisfy $t_{k-1}<l<t_{k}$ for some $k, 1 \leqq k \leqq m$. Then

$$
\frac{\left|v_{l}-v_{l-p}\right|}{\left|v_{l}\right|} \leqq\left|v_{t_{k}}-v_{t_{k}-p}\right| 2 \Delta^{4} \delta^{\left(t_{k}-l-1\right) / 3},
$$

where $\Delta=|\log \delta|$. 
Proof. By Lemma 2.1 (2) we have

$$
\begin{aligned}
\frac{\left|v_{l}-v_{l-p}\right|}{\left|v_{l-p}\right|} & =\frac{\left|v_{t_{k}}-v_{t_{k}-p}\right|}{\left|v_{l-p}\right|} \prod_{j=l}^{t_{k}-1}\left(2^{n_{j+1}-1}\left(\left|v_{j}\right|+\left|v_{j-p}\right|\right)\right)^{-1} \\
& \leqq\left|v_{t_{k}}-v_{t_{k}-p}\right|\left(2^{n_{l-p+1}-1} v_{l-p}^{2}\right)^{-1} \prod_{j=l+1}^{t_{k}-1}\left(2^{n_{j-p+1}-1}\left|v_{j-p}\right|\right)^{-1} .
\end{aligned}
$$

Since $l-p \notin \mathbb{P}$, Lemma 2.3 implies,

$$
\left(2^{n_{l-p+1}-1} v_{l-p}^{2}\right)^{-1} \leqq 2 \Delta^{4},
$$

while $j-p \notin \mathbb{P}$ implies through Lemma 2.3 and 1.1,

$$
\left(2^{n_{j-p+1}-1}\left|v_{j-p}\right|\right)^{-1} \leqq 2(8 \delta)^{1 / 2} \Delta^{2} \leqq \delta^{1 / 3},
$$

for sufficiently small $\delta_{0}>0$. This proves the assertion.

We next want to include the terms $t_{k}$ in our product.

Lemma 3.2. Let $\delta \in \mathbf{J}_{s}^{4}$, let $p \in \mathbb{P}^{\prime}$ and $p<s<t(p)$. Then

$$
\prod_{l=p+1}^{s} \frac{\left|v_{l}\right|}{\left|v_{l-p}\right|} \geqq \Delta^{-4}
$$

Proof. With $t_{k}$ defined as before, we have

$$
\prod_{l=p+1}^{s} \frac{\left|v_{l}\right|}{\left|v_{l-p}\right|}=\prod_{k=1}^{m}\left\{\frac{\left|v_{t_{k}}\right|}{\left|v_{t_{k}-p}\right|} \prod_{l=t_{k-1}+1}^{t_{k}-1} \frac{\left|v_{l}\right|}{\left|v_{l-p}\right|}\right\} \prod_{l=t_{m}+1}^{s} \frac{\left|v_{l}\right|}{\left|v_{l-p}\right|} .
$$

From $v_{l} v_{l-p}>0$ and from Lemma 3.1, we have for $t_{k-1}<l<t_{k}$,

$$
\frac{\left|v_{l}\right|}{\left|v_{l-p}\right|} \geqq 1-\frac{\left|v_{l}-v_{l-p}\right|}{\left|v_{l-p}\right|} \geqq 1-\left|v_{t_{k}}-v_{t_{k}-p}\right| 2 \Delta^{4} \delta^{\left(t_{k}-l-1\right) / 3} \text {. }
$$

Therefore,

$$
\prod_{l=t_{k-1}+1}^{t_{k}-1} \frac{\left|v_{l}\right|}{\left|v_{l-p}\right|} \geqq \prod_{n=0}^{\infty}\left(1-C \delta^{n / 3}\right),
$$

where $C=\left|v_{t_{k}}-v_{t_{k}-p}\right| 2 \Delta^{4}$. Since $1-x>\exp (-2 x)$ for $0 \leqq x \leqq \frac{1}{2}$, the above product is bounded below by $\exp \left(-2 C\left(1-\delta^{1 / 3}\right)^{-1}\right)$ provided $C<\frac{1}{2}$.

We are now going to prove $C<\frac{1}{2}$. If $t_{k} \in \mathbb{P}$, then

$$
C \leqq 2 \Delta^{4}\left(\left|v_{t_{k}}\right|+\left|v_{t_{k}-p}\right|\right) \leqq \delta^{1 / 3} \text {, }
$$

by the definition of $\mathbb{P}$ (note $t_{k}-p \in \mathbb{P}$, too), and by Lemma 1.1. If $t_{k} \notin \mathbb{P}$, we have by Lemma 2.1 (2),

$$
\left|v_{t_{k}}-v_{t_{k}-p}\right| \leqq 2 /\left(2^{n_{t_{k}}+1}\left|v_{t_{k}}\right|\right)
$$

so that by the definition of $\mathbb{P}$ and by Lemma $2.3, C \leqq \delta^{1 / 3}$ in this case. 
Summarizing, we have found so far

$$
\begin{aligned}
X_{k} & \equiv \frac{\left|v_{t_{k}}\right|}{\left|v_{t_{k}-p}\right|} \prod_{l=t_{k-1}+1}^{t_{k}-1} \frac{\left|v_{l}\right|}{\left|v_{l-p}\right|} \\
& \geqq \frac{\left|v_{t_{k}}\right|}{\left|v_{t_{k}-p}\right|} \exp \left(-5 \Delta^{4}\left|v_{t_{k}}-v_{t_{k}-p}\right|\right) .
\end{aligned}
$$

We now use Lemma 2.2. If the first alternative of its conclusion holds, then $\left|v_{t_{k}}\right|$ $>\left|v_{t_{k}-p}\right|$ and

$$
\frac{\left|v_{t_{k}}\right|}{\left|v_{t_{k}-p}\right|}=1+\frac{\left|v_{t_{k}}-v_{t_{k}-p}\right|}{\left|v_{t_{k}-p}\right|}
$$

and since $t_{k}-p \in \mathbb{P}$ we have $\left|v_{t_{k}-p}\right|^{-1} \geqq \Delta^{2} 3.3^{-1} 2^{n_{1 / 2}}$. Therefore, by Lemma 1.1,

$$
\begin{aligned}
X_{k} & \geqq\left(1+\left|v_{t_{k}}-v_{t_{k}-p}\right| \delta^{-1 / 3}\right)\left(1-5 \Delta^{4}\left|v_{t_{k}}-v_{t_{k}-p}\right|\right) \\
& \geqq 1,
\end{aligned}
$$

where the last inequality follows by simple arithmetic from $5 \Delta^{4}<\delta^{-1 / 3}$. If the second alternative of Lemma 2.2 holds, then

$$
\begin{aligned}
X_{k} & \geqq\left(1-\frac{\left|v_{t_{k}}-v_{t_{k}-p}\right|}{\left|v_{t_{k}-p}\right|}\right)\left(1-5 \Delta^{4}\left|v_{t_{k}}-v_{t_{k}-p}\right|\right) \\
& \geqq 1-\left|v_{t_{k}}-v_{t_{k}-p}\right|\left(\frac{1}{\left|v_{t_{k}-p}\right|}+5 \Delta^{4}\right) \\
& \geqq 1-\frac{1}{2}\left(t_{k}-p\right)^{-2}\left(1+5 \Delta^{4}\left|v_{t_{k}-p}\right|\right) .
\end{aligned}
$$

Since $t_{k}-p \in \mathbb{P},\left|v_{t_{k}-p}\right|<\delta^{1 / 3}$, by Lemma 1.1 , and hence

$$
X_{k} \geqq 1-\frac{2}{3}\left(t_{k}-p\right)^{-2} \text {. }
$$

We next analyze the product

$$
Y \equiv \prod_{l=t_{m}+1}^{s}\left|v_{l} / v_{l-p}\right| .
$$

We distinguish two cases.

Case 1. $s<t(p)-1$. As before, we find, of (3.3), [Lemma 3.1 holds for $t_{m+1}=t(p)$ ],

$$
\begin{aligned}
Y & \geqq \prod_{l=t_{m}+1}^{t(p)-2}\left(1-\left|v_{t(p)}-v_{t(p)-p}\right| 2 \Delta^{4} \delta^{(t(p)-l-1) / 3}\right) \\
& \geqq \prod_{n=1}^{\infty}\left(1-C \delta^{n / 6}\right) \geqq \frac{1}{2}
\end{aligned}
$$

because $C<\frac{1}{2}$ for sufficiently small $\delta_{0}$, with

$$
C=\left|v_{t(p)}-v_{t(p)-p}\right| 2 \Delta^{4} \delta^{1 / 6} \text {. }
$$

Case 2. $s=t(p)-1$, and $s-p \notin \mathbb{P}$. We use Lemma 2.4. By multiplying the result for the case $s=t(p)-2$ with the bound for $\left|v_{t(p)-1} / v_{t(p)-p-1}\right|$ we get immediately 
$Y \geqq \frac{1}{2} \Delta^{-3}$. [Note that if $s=t(p)-1$ and $s-p \in \mathbb{P}$ then $s=t_{m}$, by the definition of $t_{m}$, so that we have exhausted all cases.]

We complete now the proof of Lemma 3.2 by multiplying the bounds for $X_{k}$ and $Y$. We get

$$
\prod_{k=1}^{m} X_{k} \geqq \prod_{k=1}^{m}\left(1-\frac{2}{3}\left(t_{k}-p\right)^{-2}\right) .
$$

This product is bounded below by

$$
\prod_{j=1}^{\infty}\left(1-\frac{2}{3} j^{-2}\right) \geqq \frac{1}{3} \mathrm{e}^{-4 / 3} \geqq \frac{1}{20} .
$$

All this combined yields the desired bound.

Corollary 3.3. Let $\delta \in \mathbf{J}_{s}^{4}$ let $p \in \mathbb{P}^{\prime}$ and $p<s<t(p)$. Then

$$
\prod_{l=p+1}^{s} \frac{2\left|v_{l}\right|}{\left|v_{l}\right|+\left|v_{l-p}\right|} \geqq \Delta^{-4}
$$

Proof. Let $A, B>0$ and suppose $|A-B| / B<\varepsilon<1$. Then we have $2 A /(A+B)>1-\varepsilon$, as one can easily check. Using this inequality with $A=\left|v_{l}\right|$ and $B=\left|v_{l-p}\right|$, we find by Lemma 3.1, with the notation introduced there,

$$
W_{k} \equiv \prod_{l=t_{k-1}+1}^{t_{k}-1} \frac{2\left|v_{l}\right|}{\left|v_{l}\right|+\left|v_{l-p}\right|} \geqq \exp \left(-4 \Delta^{4}\left|v_{t_{k}}-v_{t_{k}-p}\right|\right)
$$

cf. the derivation of Eq. (3.2). If $t_{k} \neq t(p)$, then we bound

$$
W_{k}^{\prime} \equiv W_{k} 2\left|v_{t_{k}}\right| /\left(\left|v_{t_{k}}\right|+\left|v_{t_{k}-p}\right|\right)
$$

as follows. If the first alternative of Lemma 2.2 holds, then

$$
\begin{aligned}
W_{k}^{\prime} & \geqq \exp \left(-5 \Delta^{4}\left|v_{t_{k}}-v_{t_{k}-p}\right|\right) \cdot\left(1+\left|v_{t_{k}}-v_{t_{k}-p}\right| /\left(2\left|v_{t_{k}-p}\right|\right)\right) \\
& \geqq 1
\end{aligned}
$$

as in Eq. (3.3). If the second alternative of Lemma 2.2 holds, then

$$
\begin{aligned}
W_{k}^{\prime} \geqq & \exp \left(-5 \Delta^{4}\left|v_{t_{k}}-v_{t_{k}-p}\right|\right) \\
& \cdot\left(1-\left|v_{t_{k}}-v_{t_{k}-p}\right| /\left|v_{t_{k}-p}\right|\right) \\
\geqq & 1-\frac{1}{2}\left(t_{k}-p\right)^{-2}\left(1+5 \Delta^{4}\left|v_{t_{k}-p}\right|\right) .
\end{aligned}
$$

The result follows as in the proof of Lemma 3.1.

Corollary 3.4. Let $\delta \in \mathbf{J}_{s}^{4}$ let $p \in \mathbb{P}^{\prime}$, and $p<s \leqq t(p)$. Then

$$
\prod_{l=p+1}^{s} \frac{2\left|v_{l-p}\right|}{\left|v_{l}\right|+\left|v_{l-p}\right|} \leqq C
$$

for some universal constant $C$. 
Proof. Let $A, B>0$ and suppose $|A-B| / B<\varepsilon<\frac{1}{2}$. Then we have $2 B /(A+B)<1+\varepsilon$, as one can easily check. Using $A=\left|v_{l}\right|, B=\left|v_{l-p}\right|$, we find by Lemma 3.1,

$$
\stackrel{\circ}{W}_{k} \equiv \prod_{l=t_{k-1}+1}^{t_{k}-1} \frac{2\left|v_{l-p}\right|}{\left|v_{l}\right|+\left|v_{l-p}\right|} \leqq \exp \left(4 \Delta^{4}\left|v_{t_{k}}-v_{t_{k}-p}\right|\right) \text {, }
$$

cf. Eq. (3.2). The factors

$$
\stackrel{\circ}{W}_{k}^{\prime} \equiv \frac{2\left|v_{t_{k}-p}\right|}{\left|v_{t_{k}}\right|+\left|v_{t_{k}-p}\right|} \stackrel{\circ}{W}_{k}
$$

are bounded, using Lemma 2.2. If the first alternative holds, then

$$
\stackrel{\circ}{W}_{k}^{\prime} \leqq \stackrel{\circ}{W}_{k}\left(1-\frac{1}{2} \frac{\left|v_{t_{k}}-v_{t_{k}-p}\right|}{\left|v_{t_{k}-p}\right|}\right) \leqq 1,
$$

while in the second case

$$
\begin{aligned}
\stackrel{\circ}{W}_{k}^{\prime} & =\stackrel{\circ}{W}_{k}\left(1+\frac{\left|v_{t_{k}}-v_{t_{k}-p}\right|}{\left|v_{t_{k}-p}\right|}\right) \\
& \leqq 1+\left(t_{k}-p\right)^{-2}\left(\frac{1}{2}+8 \Delta^{4}\left|v_{t_{k}-p}\right|\right) \\
& \leqq 1+\frac{2}{3}\left(t_{k}-p\right)^{-2} .
\end{aligned}
$$

We may now multiply over $k$, when $s<t(p)$, as in the proof of Lemma 3.2, and the result follows in all cases [even for $s=t(p)]$, since $2\left|v_{t_{m}-p}\right| /\left(\left|v_{t_{m}}\right|+\left|v_{t_{m}-p}\right|\right) \leqq 2$, if $\delta_{0}$ is sufficiently small.

\section{Bounds on the Derivative}

We now derive recursive bounds on the quantity

$$
R_{m}=\prod_{j=1}^{m}\left|v_{j}\right| 2^{n_{j+1}}
$$

which is directly related to the derivative, cf. p. 117

Proposition 4.1. The following relations hold, when $\delta \in \mathbf{J}_{n}^{4}$ :

$$
R_{n} \geqq\left\{\begin{array}{l}
\Delta^{-4} R_{p} R_{n-p}, \\
\quad \text { if } p<n<t(p) \text { for some } p \in \mathbb{P}^{\prime}, \\
\Delta^{-8} R_{p-1} R_{t(p)-p-1}^{1 / 2} 2^{n_{1} / 2} \\
\cdot\left|v_{t(p)}-v_{t(p)-p}\right|^{1 / 2} \prod_{l=t(p)}^{n}\left|v_{l}\right| 2^{n_{l+1}}, \\
\text { if } n \geqq t(p) \text { for some } p \in \mathbb{P}^{\prime} .
\end{array}\right.
$$

Proof. The claim in the first case is equivalent to

$$
\prod_{j=p+1}^{n}\left|v_{j}\right| \geqq \Delta^{-4} \prod_{j=p+1}^{n}\left|v_{j-p}\right| .
$$

Since $n<t(p)$, this inequality is an immediate consequence of Lemma 3.2. 
Let now $n=t(p)$. In this case, the potentially small, resonant factor $v_{p}$ will disappear in the estimate and will be absorbed in the factor $R_{t(p)-p-1}^{1 / 2}\left|v_{t(p)}-v_{t(p)-p}\right|^{1 / 2}$. The effects of the resonance at $p$ are thus partially gone when the estimation has proceed to $t(p)$. From the definition of $R_{t(p)}$, we get

$$
\begin{aligned}
R_{t(p)}= & R_{p-1} 2^{n_{p+1}}\left|v_{p}\right| \prod_{j=p+1}^{t(p)} 2^{n_{j+1}}\left|v_{j}\right| \\
\geqq & R_{p-1} 2^{n_{p+1}} \frac{\left|v_{t(p)}-v_{t(p)-p}\right|^{1 / 2}}{2^{\left(n_{t(p)}-1\right) / 2}} \prod_{j=1}^{t(p)-p-1} \frac{2^{1 / 2+n_{p+j+1}}\left|v_{p+j}\right|}{2^{n_{p+j}}\left(\left|v_{p+j}\right|+\left|v_{j}\right|\right)^{1 / 2}} \\
& \cdot 2^{n_{t(p)+1}}\left|v_{t(p)}\right|,
\end{aligned}
$$

by Lemma 2.1. By Corollary 3.3, this is bounded below by

$$
\begin{aligned}
R_{t(p)} \geqq & R_{p-1} 2^{n_{p+1}} \frac{\left|v_{t(p)}-v_{t(p)-p}\right|^{1 / 2}}{2^{\left(n_{t(p)}-1\right) / 2}} \prod_{j=1}^{t(p)-p-1}\left|v_{p+j}\right|^{1 / 2} 2^{n_{p+j+1}-n_{p+j} / 2} \\
& \cdot 2^{n_{t(p)+1}\left|v_{t(p)}\right| \Delta^{-4}} \\
= & R_{p-1} R_{t(p)-p-1}^{1 / 2}\left|v_{t(p)}-v_{t(p)-p}\right|^{1 / 2} \Delta^{-4} \\
& \cdot 2^{n_{1} / 2+1 / 2}\left|v_{t(p)}\right| 2^{n_{t(p)+1}} \prod_{j=1}^{t(p)-p-1}\left|v_{p+j} / v_{j}\right|^{1 / 2} \\
\geqq & R_{p-1} R_{t(p)-p-1}^{1 / 2}\left|v_{t(p)}-v_{t(p)-1}\right|^{1 / 2} \Delta^{-8} \\
& \cdot 2^{n_{1} / 2}\left|v_{t(p)}\right| 2^{n_{t(p)+1}},
\end{aligned}
$$

by Lemma 3.2. This proves the proposition, even in the case $n \geqq t(p)-1$, which is an obvious consequence of what we have done for $n=t(p)$.

We next show that when $\delta \in \mathbf{J}_{n}^{4}$, then $R_{n}$ is large.

Proposition 4.2. Let $n \in \mathbb{N}$, let $\delta \in \mathbf{J}_{n}^{4}$ and let $p \in \mathbb{P}^{\prime}$ be such that $p \leqq n<f(p)$. Then

$$
R_{n} \geqq \delta^{-1 / 8-q(n) / 10} .
$$

If, in addition, one has $t(p) \leqq n<f(p)$, or $n=t(p)-1=f(p)-1$, then

$$
R_{n} \geqq \delta^{-1 / 8-q(n) / 8} .
$$

If $n<p_{0}$, then (4.2) holds.

Proof. Note $\delta^{-1 / 8}>\delta^{-1 / 10}$, so that (4.2) is a better bound than (4.1). First note that $q(n)>0$ when $n>0$ since from $\delta \in \mathbf{J}_{1}^{4}$ and Lemma $2.5, p_{0}>1$. We prove first the result for all $n<p_{0}$. Then we proceed by induction on $n \geqq p_{0}$, by assuming the result for all $R_{j}$ when $j<n$.

Case of $n<p_{0}$. In this case, we use the equation

$$
R_{n}=\prod_{j=1}^{n}\left|v_{j}\right| 2^{n_{j+1}} .
$$

Since $\delta \in \mathbf{J}_{n}^{4}$, we have $2^{n_{j+1}}\left|v_{j}\right|^{2} \geqq \Delta^{-4}$, by Lemma 2.5 , and hence by Lemma 1.1 , we find $R_{n} \geqq \delta^{-n / 3}$. This proves the assertion.

We claim now that there remain four cases for $n$. 
Case 1. $p \leqq n<t(p)$.

Case 2. $t(p) \leqq n<f(p)$.

Case 3. $n=t(p)-1=f(p)-1>p$.

Case 4. $n=t(p)-1=f(p)-1=p$.

That this is a complete set of possibilities is checked by inspection. We now discuss the four cases which occur in the inductive part of the proof.

Case 1. For some $p \in \mathbb{P}^{\prime}$, one has $p \leqq n<t(p)$. From Proposition 4.1 , we have

$$
\begin{aligned}
R_{n} \geqq & \Delta^{-4} R_{p} R_{n-p} \\
= & \Delta^{-4} R_{p-1} 2^{n_{1}}\left|v_{p}\right| R_{n-p} \\
\geqq & \Delta^{-4} \delta^{-1 / 8-q(p-1) / 8} \cdot \delta^{-q(n-p) / 10} \\
& \cdot 2^{n_{1}}\left|v_{p}\right| .
\end{aligned}
$$

Since $q(n)=q(p-1)$, we find

$$
\begin{aligned}
R_{n} \geqq & \delta^{-1 / 8-q(n) / 8} \\
& \cdot\left(2^{n_{1}}\left|v_{p}\right| \Delta^{-4}\right) .
\end{aligned}
$$

By Lemma 1.1, and by $\delta \in \mathbf{J}_{p}^{4}$ (which follows from $\delta \in \mathbf{J}_{n}^{4}$ ), we find from Lemma 2.5, $2^{n_{1}}\left|v_{p}\right| \geqq \delta^{-1} \Delta^{-4 p} / 8$.

Therefore we find

$$
\begin{aligned}
R_{n} \geqq & \delta^{-1 / 8-q(n) / 10} \\
& \cdot\left(\delta^{-1} 2^{-3} \Delta^{-4}\right) \\
& \cdot\left(\delta^{-q(n) / 40} \Delta^{-4 p}\right) .
\end{aligned}
$$

For sufficiently small $\delta_{0}$ the last two factors are not smaller than 1 , since $q(n)=q(p)$ $>p(1-\varepsilon)$ by Lemma 1.6. This proves the assertion in Case 1.

Case 2. $t(p) \leqq n<f(p)$ for some $p \in \mathbb{P}^{\prime}$. This is the crucial case, in which we avoid a factor $\Delta^{-4 p}$ which would occur had we not eliminated $v_{p}$ in Proposition 4.1. We use the result obtained there:

$$
\begin{aligned}
R_{n} \geqq & \Delta^{-8} R_{p-1} R_{t(p)-p-1}^{1 / 2} 2^{n_{1} / 2}\left|v_{t(p)}-v_{t(p)-p}\right|^{1 / 2} \\
& \cdot \prod_{l=t(p)}^{n}\left|v_{l}\right| 2^{n_{l+1}} .
\end{aligned}
$$

By Lemma 1.1, 2.3 and since $\delta \in \mathbf{J}_{n}^{4}$, we find $\left|v_{l}\right| 2^{n_{l+1}} \geqq \delta^{-1 / 3}$, and $2^{n_{1} / 2} \geqq \delta^{-1 / 3}$, so that

$$
\begin{aligned}
R_{n} \geqq & \Delta^{-8} R_{p-1} \delta^{-1 / 3} R_{t(p)-p-1}^{1 / 2}\left|v_{t(p)}-v_{t(p)-p}\right|^{1 / 2} \\
& \cdot 2^{n_{t(p)+1} / 2} \delta^{-(n-t(p)) / 3} \Delta^{-2} .
\end{aligned}
$$

Next we use Corollary 2.7 (note $\delta \in \mathbf{J}_{n}^{4}$ implies $\delta \in \mathbf{J}_{t(p)}^{4}$ :

$$
\left|v_{t(p)}-v_{t(p)-p}\right| \geqq 2^{-n_{t(p)+1}} \Delta^{-15(t(p)-p+1)} \text {. }
$$


Hence we find

$$
\begin{aligned}
R_{n} \geqq & R_{p-1} R_{t(p)-p-1}^{1 / 2} \delta^{-(n-t(p)+1) / 3} \\
& \cdot \Delta^{-15(t(p)-p-1)-40} .
\end{aligned}
$$

We now substitute the recursive bounds and get

$$
\begin{aligned}
R_{n} \geqq & \delta^{-1 / 8-q(p-1) / 8} \delta^{-q(t(p)-p-1) / 20} \\
& \cdot \delta^{-(n-t(p)+1) / 3} \Delta^{-15(t(p)-p-1)-40} .
\end{aligned}
$$

Since $n-t(p)+1>0$, we have $\delta^{-(n-t(p)+1) / 3} \geqq \delta^{-1 / 6-(n-t(p)+1) / 8}$. By definition, $q(p-1)+n-t(p)+1=q(n)$. Therefore, we find

$$
\begin{aligned}
R_{n} \geqq & \delta^{-1 / 8-q(n) / 8} \\
& \cdot\left(\delta^{-1 / 6} \Delta^{-40}\right) \\
& \cdot\left(\delta^{-q(t(p)-p-1) / 40} \Delta^{-15(t(p)-p-1)}\right) .
\end{aligned}
$$

Since $\delta \notin \mathbf{I}_{n}^{4}$, Lemma 1.6 holds and we can thus make each of the last two factors greater than one, by choosing $\delta_{0}$ sufficiently small.

Case 3. $n=t(p)-1=f(p)-1>p$. Hence, we have $t(p)-p-1>0$, and thus

$$
\begin{aligned}
R_{n}= & R_{t(p)} /\left(2^{n_{t(p)+1}}\left|v_{t(p)}\right|\right) \\
\geqq & \Delta^{-8} R_{p-1} R_{t(p)-p-1}^{1 / 2} 2^{n_{1} / 2}\left|v_{t(p)}-v_{t(p)-p}\right|^{1 / 2} \\
\geqq & \Delta^{-8} \delta^{-1 / 8-q(p-1) / 8} \delta^{-1 / 16-q(t(p)-p-1) / 20} \\
& \cdot 2^{n_{1} / 2-n_{t(p)+1 / 2}} \Delta^{-8(t(p)-p-1)} .
\end{aligned}
$$

In the last inequality, we have used Corollary 2.7 and $t(p)-p+1 \leqq n$, since $p>1$. We also have $q(n)=q(t(p)-1)=q(p-1)$. Since $t(p)=f(p)$, we find $n_{t(p)+1}=n_{1}$ and thus

$$
\begin{aligned}
R_{n} \geqq & \delta^{-1 / 8-q(n) / 8} \\
& \cdot\left(\delta^{-1 / 16} \Delta^{-8}\right) \\
& \cdot\left(\delta^{-q(t(p)-p-1) / 20} \Delta^{-8(t(p)-p-1)}\right) .
\end{aligned}
$$

Applying again the argument of Case 1, the last two factors in (4.3) are bounded below by 1 , and the assertion is proved in this case.

Case 4. $n=t(p)-1=f(p)-1=p$. We have

$$
\begin{aligned}
R_{n} & =R_{p-1}\left|v_{p}\right| 2^{n_{p+1}} \\
& =R_{p-1} 2^{\left(n_{1}+1\right) / 2}\left|v_{p+1}-v_{1}\right|^{1 / 2},
\end{aligned}
$$

by Lemma $2.1(3)$, and since $n_{p+1}=n_{1}$. Since $p+1=f(p) \in \mathbb{P}$, we must have $\left|v_{p+1}\right|$ $<\delta^{1 / 4}$, by Lemma 1.1. On the other hand, $\delta \in \mathbf{J}_{1}^{4} \subset \mathbf{J}_{n}^{4}$ implies $\left|v_{1}\right|>\Delta^{-4}$. Hence we find $\left|v_{p+1}-v_{1}\right|^{1 / 2}>\Delta^{-3}$. Substituting into (4.4), we get

$$
R_{n} \geqq \delta^{-1 / 8-q(p-1) / 8} 2^{n_{1 / 2}} \Delta^{-3},
$$

and since $q(n)=q(p)=q(p-1)$ we have

$$
R_{n} \geqq \delta^{-1 / 8-q(n) / 8} \cdot\left(\delta^{-1 / 3} \Delta^{-3}\right) .
$$

This last factor is larger than 1 and thus the assertion follows. 
Corollary 4.3. Let $n \in \mathbb{N}$, let $\delta \in \mathbf{J}_{n}^{4}$. Then

$$
R_{n} \geqq \delta^{-1 / 8-n / 11}(n+1)^{20} .
$$

This follows at once from Proposition 4.2 and from Lemma 1.6.

Lemma 4.4. Let $n>0$ and $\delta \in \mathbf{J}_{n}^{4}$. Let $p \in \mathbb{P}^{\prime}$ and $p \leqq n$ and $t(p)-p-1 \leqq n$. Then $t(p)-p \leqq p$.

Proof. The proof assumes Proposition 4.2 for $n$. From Lemma 2.1, we have

$$
\begin{aligned}
v_{p}^{2} & =\prod_{j=p+1}^{t(p)-1} 2^{1-n_{j}}\left(\left|v_{j}\right|+\left|v_{j-p}\right|\right)^{-1} \cdot \frac{\left|v_{t(p)}-v_{t(p)-p}\right|}{2^{n_{t(p)}-1}} \\
& \leqq 4 \prod_{j=1}^{t(p)-p-1} 2^{-n_{p+j+1}}\left|v_{j}\right|^{-1} \cdot \prod_{j=p+1}^{t(p)-1} \frac{2\left|v_{j-p}\right|}{\left|v_{j}\right|+\left|v_{j-p}\right|} .
\end{aligned}
$$

By Corollary 3.4, this is bounded by

$$
v_{p}^{2} \leqq 4 C R_{t(p)-p-1}^{-1} \text {. }
$$

By Proposition 4.2 for $s=t(p)-p-1$, this is bounded by

$$
v_{p}^{2} \leqq 4 \delta^{q(s) / 10} C \leqq \delta^{s / 11},
$$

by Lemma 1.6. On the other hand, since $p \in \mathbb{P}$, and $\delta \notin \mathbf{I}_{p}^{1}$, we have $v_{p}^{2} \geqq \Delta^{-8 p}$, so that we find

$$
-8 p \log \Delta \leqq-(t(p)-p-1) \Delta / 11,
$$

i.e. $t(p)-p \leqq p 88 \log \Delta / \Delta \leqq p$, since $p>1$ and $88 \log \Delta / \Delta<\frac{1}{3}$.

\section{Exponential Divergence}

The purpose of this section is to improve the bounds on $R_{s}$ so as to show that the characteristic exponent of the trajectory of the critical point is strictly positive.

Consider the functions $n_{i}(\delta), B_{i}(\delta)$ and $\varrho_{i}(\delta)=\operatorname{sign} v_{i}(\delta)$. It is clear from their definition that these are piecewise constant functions of $\delta$. On the other hand, fixing $n_{i}, B_{i}, \varrho_{i}$ for $i=1, \ldots, s$ defines a set $\mathbf{K}\left(\left\{n_{i}, B_{i}, \varrho_{i}\right\}_{i \leqq s}\right)$ of values of $\delta$ for which $n_{i}(\delta)=n_{i}, B_{i}(\delta)=B_{i}$ and $\varrho_{i}(\delta)=\varrho_{i}$, for $i=1, \ldots, s$. We next cast the classification of the $n_{i}, B_{i}$, and $\varrho_{i}$ into a more convenient form. Namely, we fix first the set $\mathbb{P}^{\prime}$ and the set $\left\{t(p) \mid p \in \mathbb{P}^{\prime}\right\}$. More precisely, given $\delta$, define

$$
\begin{aligned}
\Sigma_{s}(\delta)= & \{n \in \mathbb{N} \mid n \leqq s \text { and } \\
& \left.\left(n<p_{0} \text { or } t(p)<n<f(p) \text { for some } p \in \mathbb{P}^{\prime}\right)\right\},
\end{aligned}
$$

[the "unblocked" indices],

$$
T_{s}(\delta)=\left\{p \in \mathbb{N} \mid p \in \mathbb{P}^{\prime} \text { and } t(p)<f(p) \text { and } t(p) \leqq s\right\},
$$

[those $p \in \mathbb{P}^{\prime}$ for which $t(p)<f(p)$ ],

$$
T_{s}^{\prime}(\delta)=\left\{p \in \mathbb{N} \mid p \in \mathbb{P}^{\prime} \text { and } t(p)=f(p) \leqq s\right\},
$$


[those $p \in \mathbb{P}^{\prime}$ for which $\left.t(p)=f(p)\right]$,

$$
Q_{s}(\delta)=\left\{n \in \mathbb{N} \mid p \leqq n \leqq s<t(p) \text { for some } p \in \mathbb{P}^{\prime}\right\},
$$

[the last few indices, if they are "blocked"].

All possible sets $\Sigma_{s}, T_{s}, T_{s}^{\prime}$, and $Q_{s}$ can be obtained as follows.

1) Fix $l \geqq 0$, and $0<p_{0}<p_{1}<\ldots<p_{l} \leqq s$.

2) Fix $t_{j}\left(=t\left(p_{j}\right)\right)$ satisfying

$p_{j}<t_{j} \leqq p_{j+1}$ for $j=0, \ldots, l-1$ and $t_{l}>p_{l}$.

3) Now

$$
\begin{aligned}
T_{s}= & \left\{p_{j} \mid t_{j}<p_{j+1}, j<l\right\} \cup\left\{p_{l} \mid t_{l} \leqq s\right\}, \\
T_{s}^{\prime}= & \left\{p_{j} \mid t_{j}=p_{j+1}, j<l\right\}, \\
Q_{s}= & \emptyset, \text { if } s \geqq t_{l} \\
= & \left\{p_{l}, p_{l}+1, \ldots, s\right\}, \text { if } s<t_{l}, \\
\Sigma_{s}= & \{n \in \mathbb{N} \mid n \leqq s \text { and } \\
& \left.\left(n<p_{0} \text { or } t_{j}<n<p_{j+1} \text { for some } j<l \text { or } n>t_{l}\right)\right\} .
\end{aligned}
$$

As in the case of the $\mathbf{K}\left(\left\{n_{i}, B_{i}, Q_{i}\right\}_{i \leq s}\right)$, we define $\mathbf{K}\left(\Sigma_{s}, T_{s}, T_{s}^{\prime}, Q_{s}\right)$ to be the set of those $\delta$ for which $Q_{s}(\delta)=Q_{s}$ etc. The next two lemmas adapt the factor $R_{s-1}$ optimally to the form of the sets $\Sigma_{s}, T_{s}, T_{s}^{\prime}, Q_{s}$.

Lemma 5.1. If $\delta \in \mathbf{J}_{s}^{4} \cap \mathbf{K}\left(\left\{n_{i}, B_{i}, \varrho_{i}\right\}_{i \leqq s+1}\right) \cap \mathbf{K}\left(\Sigma_{s}, T_{s}, T_{s}^{\prime}, Q_{s}\right)$, then

$$
\begin{aligned}
R_{s} \geqq & \prod_{j \in \Sigma_{s}} 2^{n_{j+1}}\left|v_{j}\right| \\
& \cdot \prod_{p \in T_{s}} \Delta^{-8} 2^{n_{1} / 2} R_{t(p)-p-1}^{1 / 2}\left|v_{t(p)}-v_{t(p)-p}\right|^{1 / 2} 2^{n_{t(p)+1}}\left|v_{t(p)}\right| \\
& \cdot \prod_{p \in T_{s}^{\prime}} \Delta^{-8} 2^{n_{t(p)+1} / 2} R_{t(p)-p-1}^{1 / 2}\left|v_{t(p)}-v_{t(p)-p}\right|^{1 / 2} \\
& \cdot R\left(Q_{s}\right),
\end{aligned}
$$

where

$$
\begin{aligned}
& R\left(Q_{s}\right)=1, \quad \text { if } Q_{s}=\emptyset \\
& R\left(Q_{s}\right)=\Delta^{-4} 2^{n_{1}}\left|v_{p_{l}} \cdot \cdot \prod_{j=1}^{s-p_{l}} 2^{n_{j+1}}\right| v_{j} \mid \\
& \quad \text { if } Q_{s}=\left\{p_{l}, p_{l+1}, \ldots, s\right\} \quad \text { and } s \geqq p_{l} .
\end{aligned}
$$

Proof. We use extensively Proposition 4.1. Assume the result for $R_{p-1}$ where $p \in \mathbb{P}^{\prime}$. We are going to prove it recursively for all $s$ when $p \leqq s<f(p)$. The case $s<p_{0}$ then completes the inductive proof.

Case 1. $p \leqq s<t(p)$. Then we use first the inequality $R_{s} \geqq R_{p-1} R\left(Q_{s}\right)$. If $p$ equals $t\left(p^{\prime}\right)$ for some $p^{\prime} \in \mathbb{P}^{\prime}, \quad$ then $\Sigma_{s}=\Sigma_{p-1}=\Sigma_{p^{\prime}-1}, \quad T_{s}=T_{p-1}=T_{p^{\prime}}, \quad T_{s}^{\prime}=T_{p-1}^{\prime} \cup\left\{p^{\prime}\right\}$ 
$=T_{p^{\prime}}^{\prime} \cup\left\{p^{\prime}\right\}$. We then write

$$
\begin{aligned}
R_{p-1}= & R_{p^{\prime}} \cdot \prod_{j=p^{\prime}+1}^{p-1} 2^{n_{j+1}}\left|v_{j}\right| \\
= & \prod_{j \in \Sigma_{s}} \ldots \prod_{q \in T_{s}} \ldots \prod_{q \in T_{p^{\prime}}^{\prime}} \ldots \\
& \cdot \prod_{j=p^{\prime}}^{p-1} 2^{n_{j+1}\left|v_{j}\right|} \\
\geqq & \prod_{j \in \Sigma_{s}} \cdots \prod_{q \in T_{s}} \ldots \prod_{q \in T_{p^{\prime}}} \ldots \\
& \cdot \Delta^{-8} R_{t\left(p^{\prime}\right)-p^{\prime}-1}^{1 / 2}\left|v_{t\left(p^{\prime}\right)}-v_{t\left(p^{\prime}\right)-p^{\prime}}\right|^{1 / 2} 2^{n_{1} / 2}
\end{aligned}
$$

by an obvious variant of the second statement of Proposition 4.1. The formula (5.1) follows now in this case. If $p \neq t\left(p^{\prime}\right), \forall p^{\prime} \in \mathbb{P}^{\prime}$, then $\Sigma_{s}=\Sigma_{p-1}, T_{s}=T_{p-1}$, $T_{s}^{\prime}=T_{p-1}^{\prime}$, and the assertion follows immediately from $R_{s} \geqq R_{p-1} R\left(Q_{s}\right)$.

Case 2. $t(p) \leqq s<f(p)$. [The case $s=t(p)=f(p)$ has been handled in Case 1.] Then we write

$$
R_{s}=R_{t(p)-1} \prod_{j=t(p)}^{s} 2^{n_{j+1}}\left|v_{j}\right| .
$$

We have now

$$
\begin{gathered}
\Sigma_{s}=\Sigma_{p-1} \cup\{t(p)+1, \ldots, s\}, \\
T_{s}=T_{p} \cup\{p\}, \quad T_{s}^{\prime}=T_{p}^{\prime} .
\end{gathered}
$$

We now write

$$
R_{t(p)-1} \geqq R_{p-1} 2^{n_{1} / 2} \Delta^{-8} R_{t(p)-p-1}^{1 / 2}\left|v_{t(p)}-v_{t(p)-p}\right|^{1 / 2} .
$$

If $p \neq t\left(p^{\prime}\right)$ we are finished, since then $T_{p}=T_{p-1}$ and $T_{p}^{\prime}=T_{p-1}^{\prime}$, and the assertion follows from (5.3) and (5.4). If, on the other hand, $p=t\left(p^{\prime}\right)$ for some $p^{\prime} \in \mathbb{P}^{\prime}$, then we have $T_{p}^{\prime}=T_{p-1}^{\prime} \cup\left\{p^{\prime}\right\}$ and use instead of (5.1) the formula (5.2) for $R_{p-1}$ to arrive at the result.

Case 3. $s<p_{0}$. This case is trivial. This completes the proof of Lemma 5.1.

We next improve Lemma 5.1 as follows.

\section{Corollary 5.2. If}

$$
\delta \in \mathbf{J}_{s}^{4} \cap \mathbf{K}\left(\left\{n_{i}, B_{i}, \varrho_{i}\right\}_{i \leqq s+1}\right) \cap \mathbf{K}\left(\Sigma_{s}, T_{s}, T_{s}^{\prime}, Q_{s}\right),
$$

then

$$
\begin{aligned}
R_{s} \geqq & \prod_{j \in \Sigma_{s}} 2^{n_{j+1}}\left|v_{j}\right| \\
& \cdot \prod_{p \in T_{s}} \Delta^{-8} 2^{n_{1} / 2} R_{t(p)-p-1}^{1 / 2}\left|v_{t(p)}-v_{t(p)-p}\right|^{1 / 2} 2^{n_{t(p)+1}}\left|v_{t(p)}\right| \\
& \cdot \prod_{p \in T_{s}^{\prime}} \Delta^{-8} 2^{n_{t(p)+1} / 4} R_{t(p)-p-1}^{1 / 4} \\
& \cdot R\left(Q_{s}\right) .
\end{aligned}
$$


Proof. In view of Lemma 5.1, we only have to show that for $p \in \mathbb{P}^{\prime}$ and $t(p)=f(p)$,

$$
2^{n_{t(p)+1} / 4} R_{t(p)-p-1}^{1 / 4}\left|v_{t(p)}-v_{t(p)-p}\right|^{1 / 2} \geqq 1 .
$$

If $t(p)=p+1$ then we argue as in Case 4 of Proposition 4.2. So assume now $t(p)>p+1$. From Corollary 4.3 , we find

$$
R_{t(p)-p-1}^{1 / 4} \geqq \delta^{-1 / 32-(t(p)-p-1) / 100} .
$$

The inequality (5.5) will thus follow from

$$
\left|v_{t(p)}-v_{t(p)-p}\right|^{1 / 2} 2^{n_{t(p)+1} / 4} \geqq \Delta^{-8(t(p)-p+1)} .
$$

Note that this is an improvement over Lemma 2.6. We distinguish four cases. Let $\nu=v_{t(p)}, \mu=v_{t(p)-p}$.

Case 1. $\mu \nu<0$. Then $|v-\mu|>|\mu|>\Delta^{-4(t(p)-p)}$, by Lemma 2.5 .

Case 2. $|v / \mu| \geqq 2$, and $\mu \nu>0$. Then $|v-\mu| \geqq|\mu|$ and the result (5.6) follows as before.

Case 3. $|v / \mu| \leqq \frac{1}{2}$, and $\mu v>0$. Then $|v-\mu| \geqq \frac{1}{2}|\mu|$ and the result (5.6) follows.

Case 4. $\frac{1}{2}<|v / \mu|<2$ and $\mu v>0$. Since $t(p)=f(p) \in \mathbb{P}$ we have $n_{t(p)+1}=n_{1}$ and

$$
|v| \leqq 4 \cdot 2^{-n_{1} / 2} \Delta^{-2},
$$

and hence $|\mu| \leqq \frac{1}{2} 2^{-n_{1} / 2}$. Thus

$$
|v-\mu|^{1 / 2}=\left(\frac{\left|v^{2}-\mu^{2}\right|}{|v|+|\mu|}\right)^{1 / 2} \geqq 2^{n_{1} / 4}\left|v_{t(p)}^{2}-v_{t(p)-p}^{2}\right|^{1 / 2} .
$$

The assertion is now immediate from Lemma 2.6 and from $n_{t(p)+1}=n_{1}$.

Define now the number $N_{s}^{\prime}$ of "unblocked" steps,

$$
N_{s}^{\prime}=\sum_{j \in \Sigma_{s}} n_{j+1}+\sum_{p \in T_{s}} n_{t(p)+1} \text {. }
$$

Then we have the more convenient reformulation.

Corollary 5.3. If

$$
\delta \in \mathbf{J}_{s}^{4} \cap \mathbf{K}\left(\left\{n_{i}, B_{i}, \varrho_{i}\right\}_{i \leqq s+1}\right) \cap \mathbf{K}\left(\Sigma_{s}, T_{s}, T_{s}^{\prime}, Q_{s}\right),
$$

then

$$
R_{s} \geqq 2^{N_{s}^{\prime} / 6} \text {. }
$$

Proof. If $j \in \Sigma_{s}$, we have by Lemma 2.3,

$$
2^{n_{J}+1}\left|v_{j}\right| \geqq 2^{n_{J+1} / 2} \Delta^{-2}>2^{n_{j+1} / 3} .
$$

If $p \in T_{s}$, then we want to extract a factor $2^{n_{t(p)+1} / 4}$ from the corresponding term in Corollary 5.2. This term is

$$
\begin{aligned}
& \Delta^{-8} 2^{n_{1} / 2} R_{t(p)-p-1}^{1 / 2}\left|v_{t(p)}-v_{t(p)-p}\right|^{1 / 2} 2^{n_{t(p)+1}}\left|v_{t(p)}\right| \\
& \geqq \Delta^{-8} 2^{n_{1} / 2} R_{t(p)-p-1}^{1 / 2} \Delta^{-8(t(p)-p-1)} \Delta^{-24} 2^{n_{t(p)+1} / 4},
\end{aligned}
$$

(by the bound given at the end of the proof)

$$
\geqq 2^{n_{t(p)+1} / 4},
$$


[by Proposition 4.2, if $t(p)-p>1$ (using again $q(s)>s(1-\varepsilon)$ ), and by Lemma 1.1, if $t(p)-p=1]$.

Finally, the term $R\left(Q_{s}\right)$ is bounded below by $\Delta^{-4 s}$, using Proposition 4.2, and $\delta \in \mathbf{J}_{s}^{4}$. From card $\left(\Sigma_{s} \cup T_{s}\right)=q(s) \geqq s(1-\varepsilon)$ and from $2^{n_{j} / 1000}>\Delta$, we find

$$
\begin{aligned}
R_{s} \geqq & \prod_{j \in \Sigma_{s}} 2^{n_{j+1} / 3-n_{j+1} / 100} \cdot \prod_{p \in T_{s}} 2^{n_{t(p)+1} / 5-n_{t(p)+1} / 100} \\
& \cdot \Delta^{10 \operatorname{card}\left(\Sigma_{s} \cup T_{s}\right)} \Delta^{-4 s}
\end{aligned}
$$

and the assertion follows. We still have to provide the promised bound. We want to show [with $q=t(p)]$

$$
X \equiv\left|v_{q}-v_{q-p}\right|^{1 / 2}\left|v_{q}\right| 2^{3 n_{q+1} / 4} \geqq \Delta^{-8(q-p+2)} .
$$

Proof. Since $p \in T_{s}$, we have $t(p) \notin \mathbb{P}$. We distinguish three cases.

Case 1. $\left|v_{q}\right| \leqq\left|v_{q-p}\right| / 2$. Then

$$
\left|v_{q}-v_{q-p}\right| \geqq\left|v_{q-p}\right| / 2 \geqq\left|v_{q}\right| / 4
$$

so that $X \geqq\left(2^{n_{q+1} / 2}\left|v_{q}\right|\right)^{3 / 2} / 4 \geqq \Delta^{-7}$ since $q \notin \mathbb{P}$, by Lemma 2.3 .

Case 2. $\left|v_{q}\right|>\left|v_{q-p}\right| / 2$ and $v_{q} v_{q-p}>0$. Then

$$
\begin{aligned}
X & =2^{3 n_{q+1} / 4}\left|v_{q}^{2}-v_{q-p}^{2}\right|^{1 / 2}\left|v_{q}\right| /\left(\left|v_{q}\right|+\left|v_{q-p}\right|\right)^{1 / 2} \\
& \geqq \frac{1}{2}\left|v_{q}^{2}-v_{q-p}^{2}\right|^{1 / 2} 2^{n_{q+1} / 2} 2^{n_{q+1} / 4}\left|v_{q}\right|^{1 / 2} .
\end{aligned}
$$

The assertion follows now from $q \notin \mathbb{P}$, by Lemma 2.3 and from Lemma 2.6 (2)-(4).

Case 3. $\left|v_{q}\right|>\left|v_{q-p}\right| / 2$ and $v_{q} v_{q-p}<0$. Then $\left|v_{q}-v_{q-p}\right|>\left|v_{q}\right|$ and we proceed as in Case 1. The proof of Corollary 5.3 is complete.

Theorem 5.4. For $\delta \in \bigcap_{n=0}^{\infty} \mathbf{J}_{n}^{4} \equiv \mathbf{J}_{\infty}$, we have

(1) $2^{n_{1}} R_{s}\left|v_{s+1}\right| \geqq 2^{\left(n_{1}+N_{s}\right) / 7}$,

(2) $R_{s} \geqq 2^{N_{s} / 7}$,

where $N_{s}=\sum_{j=1}^{s} n_{j+1}$.

Proof. Fix $\delta \in \mathbf{J}_{\infty}$, and assume first, for some $p \in \mathbb{P}^{\prime}$, that $t(p)<s \leqq f(p)$, or that $s<p_{0}$. From Corollary 5.3, we have

$$
R_{s} \geqq 2^{N_{s}^{\prime} / 6} \geqq 2^{N_{s}^{\prime} / 7+(q(s)-1) \Delta / 900},
$$

since $n_{j} \geqq\left|\log _{2} \delta\right| / 2 \geqq \Delta / 3$. We rewrite this, using Lemma 2.5 , as

$$
\begin{aligned}
2^{n_{1}} \mathrm{R} & \left|v_{s+1}\right| \geqq 2^{\left(n_{1}+N_{s}^{\prime}\right) / 7+S_{s} / 7} 2^{-S_{s} / 7+(q(s)-1) \Delta / 900} \\
& \cdot 2^{n_{1} / 7} \Delta^{-4(s+1)},
\end{aligned}
$$

where $S_{s}=N_{s}-N_{s}^{\prime}$. But

$$
S_{s}=\sum_{j} b, s n_{j+1}
$$


cf. Eq. (0.5). The assertion (1) of the theorem will thus follow if

$$
0 \leqq n_{1} / 7-S_{s} / 7+(q(s)-1) \Delta / 900-\left(\log _{2} \Delta\right) 4(s+1)
$$

but this is obvious from $\delta \notin \mathbf{I}_{s}^{4}$ and from Lemma 1.6. The assertion (2) is a simpler variant and follows in the same way.

\section{The Excluded Volume}

In order to bound the excluded volume, we need a lower bound on the "speed" with which a resonant interval is traversed when $\delta$ is varied. This is done in the first part of this section. These bounds are done recursively, i.e. when we bound the excluded volume at "level" s, i.e. $\left|\mathbf{J}_{s-1}^{4} \backslash \mathbf{J}_{s}^{4}\right|$, we shall use $\delta \in \mathbf{J}_{s-1}^{4}$. Let $\mathbf{I}_{s}=\mathbf{J}_{s-1}^{4} \backslash \mathbf{J}_{s}^{4}$ if $s>0$. The volumes $\mathbf{I}_{s}^{5}$ will be bounded in Sect. 7 .

Theorem 6.1. The excluded volume, $\left|\bigcup_{s \geqq 1} \mathbf{I}_{s}\right|$, satisfies

$$
\left|\bigcup_{s \geqq 1} \mathbf{I}_{s}\right| \leqq \delta_{0} \Delta_{0}^{-1}
$$

where $\Delta_{0}=\left|\log \delta_{0}\right|$.

We first give a general bound on the derivative of $\delta$ with respect to $v_{s}$.

Proposition 6.2. Let $s \geqq 1$ and $\delta \in \mathbf{J}_{s-1}^{4}$. Then

$$
\left|\frac{d \delta}{d v_{s}}\right| \leqq\left|\frac{4 \delta}{R_{s-1} 2^{n_{1}}}\right| .
$$

Note that since by Proposition $4.2, R_{s-1}$ is large, we see that $\left|d \delta / d v_{s}\right|$ is small, i.e. $\left|d v_{s} / d \delta\right|$ is large. In other terms, the resonant interval is crossed rapidly.

Proof. We prove recursively a slightly better bound, namely

$$
\left|\frac{d \delta}{d v_{s}}\right| \leqq \frac{3 \delta}{2^{n_{1}} R_{s-1}} \exp \left(\delta^{1 / 20} \sum_{j=1}^{s-1}(j+1)^{-20}\right) .
$$

This obviously implies the bound of the proposition provided $\delta_{0}$ is sufficiently small. Since $B_{1}=\delta\left(2^{n_{1}-1}+v_{1}\right)^{-1}$, we find

$$
-\frac{d \delta}{d v_{1}}=\delta\left(2^{n_{1}-1}+v_{1}\right)^{-1}
$$

so that

$$
\delta 2^{-n_{1}} \leqq\left|\frac{d \delta}{d v_{1}}\right| \leqq 3 \delta 2^{-n_{1}} .
$$

This implies (6.1) for $s=1$. Assume now (6.1) for all $i \leqq s-1$, for some $s \geqq 2$. From the basic equation

$$
1+v_{s-1}^{2}+\frac{v_{s}}{2^{n_{s}-1}}=\frac{B_{s} 2^{n_{1}}}{B_{1} 2^{n_{s}}}\left(1+\frac{v}{2^{n_{1}-1}}\right)
$$


we find

$$
\frac{d v_{s-1}}{d v_{s}}=-\frac{1}{2^{n} v_{s-1}}\left(1-\frac{B_{s}}{B_{1} 2^{n_{s}} v_{s-1}} \frac{d v_{1}}{d v_{s-1}}\right)^{-1} .
$$

Using now the proposition for $j=s-1$, and Eq. (6.3), we get

$$
\left|\frac{d v_{1}}{d v_{s-1}}\right|=\left|\frac{d \delta}{d v_{s-1}}\right|\left|\frac{d \delta}{d v_{1}}\right|^{-1} \leqq \frac{4 \delta}{2^{n_{1}} R_{s-2}} \frac{2^{n_{1}}}{\delta} .
$$

Substituting into (6.4), we get, using Lemma 1.3,

$$
\left|\frac{d v_{s-1}}{d v_{s}}\right| \leqq\left|2^{n_{s}} v_{s-1}\left(1-X_{s}\right)\right|^{-1}
$$

where $X_{s}=12 \cdot 2^{-n_{1}} R_{s-2}^{-1}\left|v_{s-1}\right|^{-1}$. By Corollary 4.3, and from $\delta \in \mathbf{J}_{s-1}^{4}$, we find

$$
X_{s} \leqq 9 \delta^{1 / 8+(s-2) / 11} \Delta^{4 s} \leqq \frac{1}{2} \delta^{1 / 20}(s+1)^{-20},
$$

and thus $\left(1-X_{s}\right)^{-1} \leqq \exp \left(\delta^{1 / 20} /(s+1)^{20}\right)$. The Eq. (6.1) follows now for $j=s$ from

$$
\left|\frac{d \delta}{d v_{s}}\right|=\left|\frac{d \delta}{d v_{s-1}}\right|\left|\frac{d v_{s-1}}{d v_{s}}\right|
$$

by induction. This completes the proof.

We now describe in more detail the order of the exclusions. Let $\mathbf{K}_{q}=\left\{\delta \mid 2^{-q-1}\right.$ $\left.<\delta \leqq 2^{-q}\right\}$. Define $V_{s, q}^{k}$ to be the volume

$$
V_{s, q}^{k}=\int_{\delta \in \mathbf{I}_{s}^{k} \cap\left(\mathbf{J}_{s}^{4}-1 \backslash\left(\mathbf{I}_{s}^{1} \cup \ldots \cup \mathbf{I}_{s}^{k-1}\right)\right) \cap \mathbf{K}_{q}} d \delta, \quad k=1, \ldots, 4 .
$$

The sum of these volumes is evidently an upper bound on the volume excluded at step $s$, when $\delta \in \mathbf{K}_{q}$. We shall exclude the volumes in the order $\mathbf{I}_{1}^{1}, \mathbf{I}_{1}^{2}, \mathbf{I}_{1}^{3}, \mathbf{I}_{1}^{4}, \mathbf{I}_{2}^{1}, \mathbf{I}_{2}^{2}, \ldots$, i.e., when we bound the volume of $\mathbf{I}_{s}^{1}$, we assume $\delta \in \mathbf{J}_{s-1}^{4}$, when we bound $\mathbf{I}_{s}^{2}$, we assume $\delta \in \mathbf{J}_{s-1}^{4} \backslash \mathbf{I}_{s}^{1}$ etc. The estimates will, however, be presented in a different order, namely first all $V_{s, q}^{1}$, in great detail. The cases $V_{s, q}^{2}$, and $V_{s, q}^{3}, V_{s, q}^{4}$ are then variants and we present their treatment later.

Case of $V_{s, q}^{1}$

We want to express the excluded volume through a precise version of

$$
\int d \delta \cong \int d v_{s}\left|\frac{d \delta}{d v_{s}}\right| \operatorname{card}\left(v_{s}^{-1}\left(v_{s}\right)\right) .
$$

We have already seen in Proposition 6.2 how to bound $d \delta / d v_{s}$. We now bound the cardinality of $v_{s}^{-1}\left(v_{s}\right)$, i.e. the number of solutions to the equation $v_{s}(\delta)=x$, when $x \in(-1,1)$.

Recall the definition of the sets $\mathbf{K}\left(\left\{n_{i}, B_{i}, \varrho_{i}\right\}_{i \leqq s}\right)$ of p. 136. We note the following.

Lemma 6.3. The equation $v_{s}(\delta)=x$, with $x \in \mathbb{R}$ has at most $2^{\text {s }}$ solutions $\delta$ in $\mathbf{K}\left(\left\{n_{i}, B_{i}, \varrho_{i}\right\}_{i \leqq s}\right)$.

Proof. Rewrite the basic equation relating $v_{j}$ to $v_{j-1}$ as

$$
v_{j}=B_{j} \tau-2^{n_{j}-1}\left(1+v_{j-1}^{2}\right)
$$

with $\tau=1 / \delta$. Substituting recursively this expression, we see that for fixed $n_{i}, B_{i}$, $i=1, \ldots, s$, the function $v_{s}$ is a polynomial of degree $2^{s}$ in $\tau$. The assertion follows. 
Next we observe that in the exclusion of $\mathbf{I}_{s}^{1}$, for fixed $s$ we exclude $\left|v_{s}\right| \leqq \Delta^{-4 s}$ (but only if $s$ is not "blocked"). So this leads to a bound $2 \Delta^{-4 s}$ for the variation of $v_{s}$. From Proposition 6.2, Lemma 6.3, and the bound on the variation of $v_{s}$ we have

$$
\begin{aligned}
V_{s, q}^{1} & \lesssim \sum_{n_{t}, B_{l}, \varrho_{l}, i \leqq s}^{q} 2^{s} \sup _{\delta}^{*}\left[\left|\frac{d \delta}{d v_{s}}\right| 2 \Delta^{-4 s}\right] \\
& \leqq \sum_{n_{i}, B_{l}, \varrho_{l}, i \leqq s}^{q} 2^{s} \sup _{\delta} *\left[\frac{16 \delta}{2^{n_{1}} R_{s-1}} \Delta^{-4 s}\right],
\end{aligned}
$$

where sup* is the supremum over the set $\mathbf{J}_{s-1}^{4} \cap \mathbf{K}_{q} \cap \mathbf{K}\left(\left\{n_{i}, B_{i}, \varrho_{i}\right\}_{i \leqq s}\right) \cap\left\{\delta \mid s \leqq p_{0}\right.$ or $\exists p \in \mathbb{P}^{\prime}$ such that $\left.t(p) \leqq s \leqq f(p)\right\}$, and $\sum^{q}$ is only over those $B_{i}$ compatible with $n_{i}$ and some $\delta \in \mathbf{K}_{q}$.

We proceed now to the correct description of the exclusion. The following observations are very crucial. The numbers $B_{j+1}, n_{j+1}, j=0,1, \ldots, s-1$ are uniquely determined by the $B_{i+1}, n_{i+1}$ with $i \in \Sigma_{s-1} \cup T_{s-1} \cup\{0\}$. Similarly, by the condition $\mathbf{T}(.,$.$) in the definition of t(p)$, the $\varrho_{j+1}, \mathrm{j}=0,1, \ldots, s-1$, are uniquely determined by the $\varrho_{i+1}$, with $i \in \Sigma_{s-1} \cup T_{s-1} \cup T_{s-1}^{\prime} \cup\{0\}$. Therefore, we may write the precise version of the previous estimate as

$$
\begin{aligned}
& V_{s, q}^{1} \leqq \sum_{Q_{s-1}, T_{s-1}, T_{s-1}^{\prime}, \Sigma_{s-1}} \sum_{\substack{B_{l+1}, n_{l+1} \\
i \in \Sigma_{s-1} \cup T_{s-1} \cup\{0\}}}^{q} \sum_{\substack{\varrho_{l+1} \\
i \in \Sigma_{s-1}}}^{q T_{s-1} \cup T_{s-1}^{\prime} \cup\{0\}} \\
& \cdot 2^{s} \sup _{\delta} *\left[\frac{16 \delta}{2^{n_{1}} R_{s-1}} \cdot \Delta^{-4 s}\right],
\end{aligned}
$$

where sup* extends over $\mathbf{J}_{s-1}^{4} \cap \mathbf{K}_{q} \cap \mathbf{K}\left(\Sigma_{s-1}, T_{s-1}, T_{s-1}^{\prime}, Q_{s-1}\right) \cap \mathbf{K}\left(\left\{n_{i}, B_{i}\right.\right.$, $\left.\left.\varrho_{i}\right\}_{i \leqq s}\right) \cap\left\{\delta \mid s \leqq p_{0}\right.$ or $\exists p \in \mathbb{P}^{\prime}$ such that $\left.t(p) \leqq s \leqq f(p)\right\}$. Substituting the bound of Corollary 5.2, we have

$$
\begin{aligned}
V_{s, q}^{1} \leqq & \mathcal{O}(1) 2^{-q} \sum_{Q_{s-1}, T_{s-1}, T_{s-1}^{\prime}, \Sigma_{s-1}} \sum_{\substack{B_{i+1}, n_{t+1} \\
i \in \Sigma_{s-1} \cup T_{s-1} \cup\{0\}}}^{q} \sum_{i \in \Sigma_{s-1} \cup T_{s-1}^{Q_{+}+1} \cup T_{s-1}^{\prime} \cup\{0\}} \sup _{\delta}^{*} 2^{s} 2^{-n_{1}} \\
& \cdot\left(\prod_{j \in \Sigma_{s-1}} 2^{-n_{j}+1}\left|v_{j}\right|^{-1}\right) \\
& \cdot\left(\left.\prod_{p \in T_{s-1}} \Delta^{8} 2^{-n_{1} / 2} R_{t(p)-p-1}^{-1 / 2}\left|v_{t(p)}-v_{t(p)-p}\right|^{-1 / 2} 2^{-n_{t(p)+1} \mid v_{t(p)}}\right|^{-1}\right) \\
& \cdot\left(\prod_{p \in T_{s-1}^{\prime}} \Delta^{8} 2^{-n_{t(p)+1} / 4} R_{t(p)-p-1}^{-1 / 4}\right)^{-4 s} R\left(Q_{s-1}\right)^{-1} .
\end{aligned}
$$

Lemma 6.4. For those $\delta$ which occur in $V_{s, q}^{1}$, one has $R\left(Q_{s-1}\right) \geqq 1$.

Proof. In order to be able to estimate $R\left(Q_{s-1}\right)$, we consider four cases.

Case 1. $s=t\left(p^{\prime}\right)=f\left(p^{\prime}\right)>p^{\prime}-1$. Then we have

$$
\begin{aligned}
R\left(Q_{s-1}\right) & =\Delta^{-4} 2^{n_{1}}\left|v_{p^{\prime}}\right| R_{s-p^{\prime}-1} \\
& \geqq \Delta^{-4} 2^{n_{1}} R_{s-p^{\prime}-1} \frac{\left|v_{s}-v_{s-p^{\prime}}\right|^{1 / 2}}{2^{\left(n_{s}-1\right) / 2}} \prod_{j=1}^{s-p^{\prime}-1} 2^{\left(1-n_{p^{\prime}+j}\right) / 2}\left(\left|v_{p^{\prime}+j}\right|+\left|v_{j}\right|\right)^{-1 / 2},
\end{aligned}
$$

by Lemma 2.1. By Corollary 3.3,

$$
\prod_{j=1}^{s-p^{\prime}-1} \frac{2^{1 / 2}}{\left(\left|v_{p^{\prime}+j}\right|+\left|v_{j}\right|\right)^{1 / 2}} \geqq \Delta^{-4} \prod_{j=1}^{s-p^{\prime}-1}\left|v_{j}\right|^{-1 / 2} .
$$


Therefore,

$$
\begin{aligned}
R\left(Q_{s-1}\right) & \geqq \Delta^{-8} 2^{n_{1} / 2}\left|v_{s}-v_{s-p^{\prime}}\right|^{1 / 2} R_{s-p^{\prime}-1} \cdot \prod_{j=1}^{s-p^{\prime}-1} 2^{-n_{j+1} / 2}\left|v_{j}\right|^{-1 / 2} \\
& \geqq \Delta^{-8} 2^{n_{1} / 2}\left|v_{s}-v_{s-p^{\prime}}\right|^{1 / 2} R_{s-p^{\prime}-1}^{1 / 2} .
\end{aligned}
$$

We now apply Corollary 2.7 and notice that $n_{s+1}=n_{1}$ since $t\left(p^{\prime}\right)=f\left(p^{\prime}\right)=s$. Therefore we deduce the bound

$$
R\left(Q_{s-1}\right) \geqq \Delta^{-8} \Delta^{-8\left(s-p^{\prime}+1\right)} R_{s-p^{\prime}-1}^{1 / 2} .
$$

We now use Corollary 4.3 and Lemma 1.1 to obtain the estimate

$$
\begin{aligned}
R\left(Q_{s-1}\right) & \geqq \delta^{-1 / 16-\left(s-p^{\prime}-1\right) / 22} \Delta^{-8\left(s-p^{\prime}+1\right)} \\
& \geqq \delta^{-1 / 16} \geqq 1 .
\end{aligned}
$$

Case 2. $t\left(p^{\prime}\right)<s \leqq f\left(p^{\prime}\right)$. We have in this case $s-1 \geqq t\left(p^{\prime}\right)$, therefore $Q_{s-1}=\emptyset$ and $R\left(Q_{s-1}\right)=1$.

Case 3. $s=t\left(p^{\prime}\right) \neq f\left(p^{\prime}\right)$. As in Case 1 , we have

$$
R\left(Q_{s-1}\right) \geqq \Delta^{-8} 2^{n_{1} / 2}\left|v_{s}-v_{s-p^{\prime}}\right|^{1 / 2} R_{s-p^{\prime}-1}^{1 / 2} .
$$

We have

$$
\left|v_{s}\right| \leqq \Delta^{-4 s} \leqq \Delta^{-4\left(s-p^{\prime}\right)-4} \leqq \Delta^{-4}\left|v_{s-p^{\prime}}\right|,
$$

by Lemma 2.5 . Therefore

$$
\left|v_{s}-v_{s-p^{\prime}}\right| \geqq\left(1-\Delta^{-4}\right)\left|v_{s-p^{\prime}}\right| \geqq \Delta^{-4\left(s-p^{\prime}+1\right)} .
$$

From this we deduce using Proposition 4.2, that

$$
R\left(Q_{s-1}\right) \geqq \Delta^{-8} \delta^{-1 / 3} \delta^{-\left(s-p^{\prime}-1\right) / 11} \Delta^{-2\left(s-p^{\prime}+1\right)} \geqq 1
$$

by the exclusion $\delta \notin \mathbf{I}_{t\left(p^{\prime}\right)-p^{\prime}}^{4}$.

Case 4. $s=t\left(p^{\prime}\right)=f\left(p^{\prime}\right)=p^{\prime}+1$. In this case we use $\left|v_{1}-v_{s}\right|>\Delta^{-5}$ and proceed as in Case 3. This completes the proof of the lemma.

Hence we now have

$$
\begin{aligned}
& V_{s, q}^{1} \leqq \mathcal{O}(1) 2^{-q} \sum_{Q_{s-1}, T_{s-1}, T_{s-1}^{\prime}, \Sigma_{s-1}} \sum_{\substack{B_{i+1}, n_{i+1} \\
i \in \Sigma_{s-1} \cup T_{s-1} \cup\{0\}}}^{\sum^{-4 s-10}} q^{-10}
\end{aligned}
$$

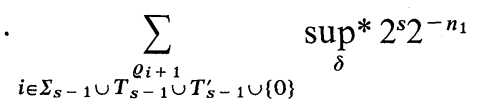

$$
\begin{aligned}
& \cdot\left(\prod_{j \in \Sigma_{s-1}} 2^{-n_{j}+1}\left|v_{j}\right|^{-1}\right) \cdot\left(\prod_{p \in T_{s-1}} \Delta^{8} 2^{-n_{1} / 2} \delta^{(t(p)-p-1) / 22}\right. \\
& \left.\cdot(t(p)-p)^{-10}\left|v_{t(p)}-v_{t(p)-p}\right|^{-1 / 2}\left|v_{t(p)}\right|^{-1} 2^{-n_{t(p)+1}}\right) \\
& \cdot\left(\prod_{p \in T_{s-1}^{\prime}} 2^{-n_{1} / 4} \Delta^{8} \delta^{(t(p)-p-1) / 44}(t(p)-p)^{-5}\right) \cdot
\end{aligned}
$$


We now apply Lemma A.3 and A.4 to sum over $n_{i}, B_{i}$, and $\varrho_{i}, i>1$. From now on, the variable $\delta$ in sup* has disappeared, and we use the symbols $\delta$ and $\Delta$ to denote their worst possible values when $\delta \in \mathbf{K}_{q}$. We obtain, using Lemma 1.6 to absorb the factor $2^{s}$,

$$
\begin{aligned}
V_{s, q}^{1} \leqq & \mathcal{O}(1) \delta \sum_{Q_{s-1}, T_{s-1}, T_{s-1}^{\prime}, \Sigma_{s-1}} \Delta^{-4\left(\left|\Sigma_{s-1} \cup T_{s-1}\right|+1\right)} \\
& \cdot 2^{2\left|\Sigma_{s-1} \cup T_{s-1} \cup T_{s-1}^{\prime}\right|+2} 2^{2\left|\Sigma_{s-1} \cup T_{s-1}\right|+2} \\
& \cdot \sum_{B_{1}, n_{1}}^{q} \cdot 2^{-n_{1}} \cdot C^{\left|\Sigma_{s-1}\right|} \\
& \cdot C^{\left|T_{s-1}\right|} \Delta^{18\left|T_{s-1}\right| 2^{-n_{1}\left|T_{s-1}\right| / 2}} \\
& \cdot \prod_{p \in T_{s-1}} \Delta^{-10(t(p)-p)} \delta^{(t(p)-p-1) / 22}(t(p)-p)^{-10} \\
& \cdot \Delta^{8\left|T_{s-1}^{\prime}\right|} 2^{-\left|T_{s-1}^{\prime}\right| n_{1} / 4} \prod_{p \in T_{s-1}^{\prime}} \delta^{(t(p)-p-1) / 44}(t(p)-p)^{-5} .
\end{aligned}
$$

From Lemma 1.1 we deduce

$$
\begin{gathered}
V_{s, q}^{1} \leqq \\
\mathcal{O}_{Q_{s-1}, T_{s-1}, T_{s-1}^{\prime}, \Sigma_{s-1}} \sum_{B_{1}, n_{1}}^{q} 2^{-n_{1}} \cdot 2^{-n_{1}\left|T_{s-1} \cup T_{s-1}^{\prime}\right| / 8} \\
\cdot \Delta^{-3\left|\Sigma_{s-1} \cup T_{s-1} \cup T_{s-1}^{\prime}\right|-3} \prod_{p \in T_{s-1} \cup T_{s-1}^{\prime}}(t(p)-p)^{-5} .
\end{gathered}
$$

We now reexpress the sum over the choices of $Q_{s-1}, T_{s-1}, T_{s-1}^{\prime}, \Sigma_{s-1}$, as explained at the beginning of Section 5 . We also use the estimate $\delta^{1 / 1000} \Delta \leqq 1$ in some cases to eliminate some factors $\Delta$. In this way we end up with

$$
\begin{aligned}
\sum_{s>0} V_{s, q}^{\prime} \leqq & \sum_{l \geqq 0} \sum_{0<p_{0}<\ldots<p_{l}} \sum_{B_{1}, n_{1}}^{q} 2^{-n_{1}} \\
& \cdot \sum_{p_{j}<t_{j} \leqq p_{j+1}, j<l} \delta \Delta^{-1}\left(t_{l}-p_{l}\right)^{-2} \\
& \cdot \prod_{j=0}^{l-1}\left(t_{j}-p_{j}\right)^{-2} \cdot 2^{-n_{1}\left|T_{s-1} \cup T_{s-1}^{\prime}\right| / 8} \Delta^{-2\left|\Sigma_{s-1}\right|} .
\end{aligned}
$$

Note now that $\left|\Sigma_{s-1}\right|=\left(p_{0}-1\right)+\left(p_{1}-t_{0}\right)+\ldots+\left(p_{l}-t_{l-1}\right)$. Summing over the $t_{j}-p_{j}$, and over $p_{j}-t_{j-1}$ and over $p_{0}$, we get with some constant $K$,

$$
\sum_{s>0} V_{s, q}^{1} \leqq \sum_{l \geqq 0} \sum_{B_{1}, n_{1}}^{q} 2^{-n_{1}} \delta \Delta^{-1} 2^{-q l / 4} K^{l+1} .
$$

From the inequalities

$$
2^{-q} \geqq B_{1}\left(2^{n_{1}-1}+v\right) \geqq 2^{-q-1}, \quad \forall v \in(-1,1),
$$

we deduce

$$
2^{n_{1}-q+1} \geqq B_{1} \geqq 2^{n_{1}-1-q-2}
$$

so that

$$
\sum_{B_{1}, n_{1}}^{q} 2^{-n_{1}} \leqq 2^{-q+1}
$$


We may now sum over $q$ and $l$ to get the statement of Theorem 6.1 in the case of the $V_{., q}^{1}$.

\section{Bound on $V_{s, q}^{2}$}

We have again a bound of the form of (6.7), but with the factor $\Delta^{-4 s}$ replaced by $\Delta^{-15 s}$ and with sup* extending over the set

$$
\begin{aligned}
\delta \in\left(\mathbf{J}_{s-1}^{4} \backslash \mathbf{I}_{s}^{1}\right) & \cap \mathbf{K}_{q} \cap \mathbf{K}\left(\Sigma_{s-1}, T_{s-1}, T_{s-1}^{\prime}, Q_{s-1}\right) \\
& \cap \mathbf{K}\left(\left\{n_{i}, B_{i}, \varrho_{i}\right\}_{i \leqq s}\right) .
\end{aligned}
$$

We again estimate $R\left(Q_{s-1}\right)$ from below. If $Q_{s-1}=\emptyset$, we have $R\left(Q_{s-1}\right)=1$. If $Q_{s-1} \neq \emptyset$, we have

$$
R\left(Q_{s-1}\right) \geqq \Delta^{-4} 2^{n_{1}}\left|v_{p_{l}}\right| R_{s-p_{l}-1},
$$

for some $p_{l}, p_{l}<s$. From Lemma 1.1, Lemma 4.2, and from $\delta \in \mathbf{J}_{p_{l}}^{4}$, we deduce

$$
R\left(Q_{s-1}\right) \geqq \Delta^{-p_{l} 5}\left(s-p_{l}\right)^{20} \text {. }
$$

Therefore we have from $s \geqq p_{l}$,

$$
\Delta^{-10 s} R\left(Q_{s-1}\right)^{-1} \leqq\left(s-p_{l}\right)^{-5} \text {. }
$$

The bound for $V_{s, q}^{2}$ is now the same as that of $V_{s, q}^{1}$, apart from a factor $\left(s-p_{l}\right)^{-5}$ if $p_{l}<s \leqq t\left(p_{l}\right)$. The end of the estimation follows as before.

Bound of $V_{s, q}^{3}$

In this case we eliminate those $\delta$ for which

$$
\begin{aligned}
& \left|\delta\left(1+v_{s}^{2}+\varepsilon 2^{-n+1}\right)-B 2^{-n+1}\right| \\
& \leqq 4 \delta 2^{-n} \Delta^{-15 s-3} \equiv \varrho
\end{aligned}
$$

with $\varepsilon= \pm 1$. Consider now the function

$$
x(v)=\delta(v)\left(1+v^{2}+\varepsilon 2^{-n+1}\right) .
$$

We want to exclude an interval of radius $\varrho$ around $x=B / 2^{n-1}$. To bound it, we first compute

$$
\frac{d x}{d v}=2 \delta(v) v+\frac{d \delta}{d v}\left(1+v^{2}+\varepsilon 2^{-n+1}\right) .
$$

By Propositions 6.2 and 5.2, we have, since $v=v_{s}$ and since we assume already $\delta \in \mathbf{J}_{s-1}^{4} \backslash\left(\mathbf{I}_{s}^{1} \cup \mathbf{I}_{s}^{2}\right)$ in the inductive process,

$$
\left|\frac{d \delta}{d v_{s}}\right| \cdot\left|v_{s}\right|^{-1} \leqq 4 \delta \delta^{(s-1) / 11+1 / 8} \Delta^{5 s} \text {. }
$$

Therefore

$$
\frac{d x}{d v_{s}}=\delta\left(v_{s}\right) v_{s}\left(2+\mathcal{O}\left(\delta^{1 / 100}\right)\right) .
$$


The corresponding $v_{s}$ intervals which are excluded through $\mathbf{I}_{s}^{3}$ have therefore a total length of at most

$$
\begin{aligned}
2 \cdot 2 \cdot \varrho \cdot\left|\frac{d v_{s}}{d x}\right| & \\
& \leqq 16 \cdot 2^{-n} \Delta^{-15 s-3}\left|v_{s}\right|^{-1} \\
& \leqq 2^{-n} \Delta^{-10 s} .
\end{aligned}
$$

We next discuss the number of solutions of $\left(1+v_{s}^{2}+\varepsilon 2^{-n+1}\right)=y \in \mathbb{R}$. This is again a polynomial equation. The function $v_{s}$ is a polynomial of degree $2^{s}$ in $\tau=1 / \delta$. Hence the whole equation is of degree $2 \cdot 2^{s}+1$ in $\tau$, i.e. for every $B, n$, there are at most $2 \cdot 2^{s}+1$ intervals of length $2^{-n} \Delta^{-10 s}$ which will be excluded. The bound for $V_{s, q}^{3}$ is therefore

$$
V_{s, q}^{3} \leqq \sum_{\substack{n_{l}, B_{l}, \varrho_{l} \\ i \leqq s}}^{q} \sum_{B, n} 2^{s+2} \sup _{\delta} *\left[\frac{16 \delta}{2^{n_{1}} R_{s-1}} 2^{-n} \Delta^{-9 s}\right] .
$$

We first perform the summation over $B$ and $n$. Since $B$ has to be compatible with $n$ and $\delta$, we obtain

$$
\sum_{B, n} 2^{-n} \leqq \sum_{n} 2^{-q} 2^{-n 2^{-q}} \leqq \mathcal{O}(1),
$$

by Lemma A.2. The estimate is now similar to that of $V_{s, q}^{2}$.

\section{Bound of $V_{s, q}^{4}$}

We recall that $N_{s}=\sum_{j=1}^{s} n_{j+1}, \mathbf{I}_{s}^{4}$ is the set of those $\delta$ for which

$$
S_{s} \equiv \sum_{j} n_{j+s} \geqq 10000(q(s)+1) \log _{2} \Delta .
$$

From the definition of $\mathbf{I}_{s}^{4}$, we deduce that there is an exclusion at $t\left(p^{\prime}\right)$, where $p^{\prime}$ is the largest element of $\mathbb{P}^{\prime}$ less than $s$. We want to exclude those $\delta$ for which $S_{t\left(p^{\prime}\right)}$ is so large that (6.8) is satisfied. We have to exclude at most a $v_{t\left(p^{\prime}\right)}$ interval of length 2 , i.e. $v_{t\left(p^{\prime}\right)} \in(-1,+1)$. As before we give an estimate on the measure of the set of those $\delta$ for which a $S_{t\left(p^{\prime}\right)}$ is too large in the preceding sense. The bound on $V_{s, q}^{4}$ is therefore:

$$
V_{s, q}^{4} \leqq \sum_{\substack{n_{l}, B_{l}, \varrho_{\imath} \\ i \leqq s}}^{2^{s}} 2^{s} \sup _{\substack{\delta:(6.8) \text { holds } \\ \text { and }\left[s=t\left(p^{\prime}\right) \\ \text { for some } p^{\prime} \in \mathbb{P}^{\prime}\right]}}\left[\frac{16 \delta}{2^{n_{1}} R_{s-1}}\right]
$$

When $s=t\left(p^{\prime}\right)$, we have from Lemma 4.3 and Lemma 1.1,

$$
\begin{aligned}
R\left(Q_{s-1}\right) & =R\left(Q_{t\left(p^{\prime}\right)-1}\right)=\Delta^{-4} 2^{n_{1}}\left|v_{p^{\prime}}\right| R_{s-p^{\prime}-1} \\
& \geqq \Delta^{-4} 2^{n_{1}} \Delta^{-4 p^{\prime}}\left(s-p^{\prime}\right)^{10} 2^{N_{s-p^{\prime}-1 / 22}} \\
& \geqq \Delta^{-5 p^{\prime}}\left(s-p^{\prime}\right)^{10} \delta^{N_{s-p^{\prime}-1 / 22}} .
\end{aligned}
$$


From Corollary 5.2 we deduce

$$
\begin{aligned}
R_{s-1} \geqq & \prod_{j \in \Sigma_{s-1}} 2^{n_{j+1}}\left|v_{j}\right| \\
& \cdot \prod_{p \in T_{s-1}} \Delta^{-8} 2^{n_{1} / 4} R_{t(p)-p-1}^{3 / 8}\left|v_{t(p)}-v_{t(p)-p}\right|^{1 / 2}\left|v_{t(p)}\right| 2^{n_{t(p)+1}} \\
& \cdot \prod_{p \in T_{s-1}^{\prime}} \Delta^{-8} 2^{n_{1} / 8} R_{t(p)-p-1}^{1 / 8} \\
& \cdot \prod_{p \in T_{s-1} \cup T_{s-1}^{\prime}} 2^{n_{1} / 8} R_{t(p)-p-1}^{1 / 8} \cdot \Delta^{-5 p^{\prime}}\left(s-p^{\prime}\right)^{10} 2^{N_{s-p^{\prime}-1}} .
\end{aligned}
$$

From Theorem 5.4 (2), we deduce

$$
\sum_{p \in T_{s-1} \cup T_{s-1}^{\prime}} 2^{n_{1} / 8} R_{t(p)-p-1}^{1 / 8} \geqq \sum_{p \in T_{s-1} \cup T_{s-1}^{\prime}} 2^{N_{t(p)-p-1 / 56}} .
$$

Note now that every blocked $n_{j+1}$ satisfies $n_{j+1}=n_{j+1-p}$. Since from the hypothesis, we have $S_{t\left(p^{\prime}\right)} / 200 \geqq 50 \log _{2} \Delta(q(s)+1)$, we obtain

$$
\Delta^{-5 p^{\prime}}\left(t\left(p^{\prime}\right)-p^{\prime}\right)^{10} 2^{N_{t\left(p^{\prime}\right)-p^{\prime}-1 / 22}} \prod_{p \in T_{s-1} \cup T_{s-1}^{\prime}} 2^{n_{1} / 8} R_{t(p)-p-1}^{1 / 8} \geqq \Delta^{15 s}\left(t\left(p^{\prime}\right)-p^{\prime}\right)^{10} .
$$

The end of the estimate is now as in the case of $V_{s, q}^{3}$.

\section{Sensitive Dependence on Initial Conditions}

In this section, we use the information obtained in Sect. 5 and the theory of Guckenheimer to establish that $f_{\delta}$ has sensitivity with respect to initial conditions, and that it is conjugate to a piecewise linear map, $g_{\tau}$; i.e. for some homeomorphism $h$, we have $h \circ f_{\delta}=g_{\tau} \circ h$.

Let $\mathbf{J}_{\infty}=\bigcap_{k=0}^{\infty} \mathbf{J}_{k}^{4}$, i.e. the set of $\delta$ retained so far, and we have shown in Sect. 6 that the Lebesgue measure of $\mathbf{J}_{\infty}$ is at least $\delta_{0}\left(1-\left|\log \delta_{0}\right|^{-1}\right)$. We shall exclude from $\mathbf{J}_{\infty}$ a further set $\bigcup_{m=1}^{\infty} \mathbf{I}_{m}^{5}$ which we define below, and whose Lebesgue measure is zero. Then the set $\mathbf{J}\left(\delta_{0}\right)$ described in the introduction is $\mathbf{J}\left(\delta_{0}\right)=\mathbf{J}_{\infty} \backslash \bigcup_{m=1}^{\infty} \mathbf{I}_{m}^{5}$ and has measure $\delta_{0}\left(1-\left|\log \delta_{0}\right|^{-1}\right)$, as claimed in Theorem A.

Theorem 7.1 If $\delta \in \mathbf{J}_{\infty}, f_{\delta}$ has no stable periodic orbit.

Proof. Assume $f$ has a stable periodic orbit $P$. Since $S\left(f_{\delta}\right)=\frac{f_{\delta}^{\prime \prime \prime}}{f_{\delta}^{\prime}}-\frac{3}{2}\left(\frac{f_{\delta}^{\prime \prime}}{f_{\delta}^{\prime}}\right)^{2} \leqq 0$, if $x \neq \frac{1}{2} \pm \delta$, we deduce from a result of [4] that $x=\frac{1}{2}$ is contained in the basin of attraction of $P$. However this contradicts our preceding result $\left|f^{n^{\prime}}\left(f_{\delta}\left(\frac{1}{2}\right)\right)\right| \rightarrow \infty$ as $n \rightarrow \infty$. We define $\{a, b\}_{ \pm}$for $a, b \in \mathbb{R}$ as $[a, b]$ if $a<b$ and $[b, a]$ if $b<a$. We now repeat some definitions of [3].

Definition. The fixed point $p$ of $f_{\delta}^{n}, n>1$, is called central if $f^{n^{\prime}}(p)>1$ and $f_{\delta}^{n}$ is a homeomorphism on the interval $J=\left\{p, \frac{1}{2}\right\}_{ \pm}$. The central point $p$ is called restrictive if $f_{\delta}^{n}(J) \subset\left\{p, p^{\prime}\right\}_{ \pm}$, where $p^{\prime}=1-p$. The point $x=0$ is not considered a central point. We also repeat two results of Guckenheimer [3]. 
Theorem 7.2. If $f_{\delta}$ has no stable periodic orbit, then $f_{\delta}$ has sensitivity to initial conditions if $f_{\delta}$ does not have a restrictive central point. (Cf. Theorem B3.)

Theorem 7.3. If $f_{\delta}$ has no stable periodic orbit, and no restrictive central point, then there is a $\left.\tau \in\left(2^{1 / 2}, 2\right)\right]$ such that $f_{\delta}$ is topologically equivalent to the map $g_{\tau}$ defined by $g_{\tau}(x)=\tau / 2-\tau\left|\frac{1}{2}-x\right|$.

Proposition 7.4. The set of preimages of $x=\frac{1}{2}$ is dense in $[0,1]$ if $\delta \in J_{\infty}$.

Proof. By [3, Theorem 2.6], since $f_{\delta}$ has no stable periodic orbit by Theorem 7.1, there is no non-trivial interval $J$ on which $\left.f_{\delta}^{n}\right|_{J}$ is a homeomorphism for all $n$. Therefore

$$
\bigcup_{n}\left\{x \mid f_{\delta}^{n}(x)=\frac{1}{2}\right\}
$$

is dense in $[0,1]$.

We now define some subsets of $\mathbf{J}_{\infty}$. For $m, k$, and $q$ in $\mathbb{N}, q \geqq q_{0}=\left[\log _{2} \delta_{0}^{-1}\right]$, we set

$$
\begin{aligned}
& \mathbf{I}_{m, q, k}^{5}=\left\{\delta \mid \inf \left(\delta_{0}, 2^{-q}\right) \geqq \delta>2^{-q-1} \quad \text { and } \quad \delta \in \mathbf{J}_{\infty} \quad\right. \text { and } \\
& \left.\{m, 2 m, \ldots, k m\} \subset\left\{\sum_{i=1}^{j} n_{i}\right\}_{j=1}^{\infty}\right\}, \\
& \mathbf{I}_{m, q}^{5}=\left\{\begin{array}{l}
\bigcap_{k=1}^{\infty} \mathbf{I}_{m, q, k}^{5}, \quad \text { if } m<2^{q / 2}, \\
\left\{\delta \mid f_{\delta} \text { has a restrictive central point of period } m\right\} \cap \mathbf{J}_{\infty} \quad \text { if } m \geqq 2^{q / 2},
\end{array}\right. \\
& \mathbf{L}_{m, k}^{5}=\bigcup_{q=q_{0}}^{\infty} \mathbf{I}_{m, q, k}^{5} \text { and } \mathbf{I}_{m}^{5}=\bigcup_{q=q_{0}}^{\infty} \mathbf{I}_{m, q}^{5} .
\end{aligned}
$$

We recall that $\mathbf{J}\left(\delta_{0}\right)=\mathbf{J}_{\infty} \backslash \bigcup_{m=1}^{\infty} \mathbf{I}_{m}^{5}$, and $E_{\delta}=\left(\frac{1}{2}-\delta, \frac{1}{2}+\delta\right)$, and $\bar{E}_{\delta}$ its closure. We now define two integers $M$ and $N$ which are functions of $\delta . M$ is the unique integer such that $\frac{1}{2} \in\left[\delta+2^{M-1} \delta, \delta+2^{M} \delta\right) ; N$ is the smallest integer such that there is a point $y \in\left[\frac{1}{2}-\delta, \frac{1}{2}\right)$ such that $f^{N}(y)=\frac{1}{2}$ [notice that by symmetry, $f^{N}(1-y)=\frac{1}{2}$ ].

\section{Lemma 7.5}

(1) If $\frac{1}{2}-\delta \leqq 2^{M} \delta<\frac{1}{2}$, then $N=M+1$.

(2) If $\frac{1}{2} \leqq 2^{M} \delta<1-2 \delta$, then $N=M$.

Proof. We first remark that as long as $f_{\delta}^{n}\left(\bar{E}_{\delta}\right) \cap \bar{E}_{\delta}=\emptyset$ and $1<n<N$, we have

$$
f_{\delta}^{n+1}\left(\bar{E}_{\delta}\right)=\left[f_{\delta}^{n+1}\left(\frac{1}{2}\right), 2^{n+1} \delta\right]=\left[2^{n} \delta, 2^{n+1} \delta\right] .
$$

(1) In this case, we deduce

$$
f_{\delta}^{M+1}\left(\bar{E}_{\delta}\right)=\left[2^{M} \delta, 1-\delta-\delta^{-1}\left(\frac{1}{2}-2^{M} \delta\right)^{2}\right] .
$$

Therefore $\frac{1}{2} \in f_{\delta}^{M+1}\left(\bar{E}_{\delta}\right)$, and we have $N=M+1$ since $f_{\delta}^{n}\left(\bar{E}_{\delta}\right) \cap \bar{E}_{\delta}=\emptyset$ if $n \leqq M-1$, and $\frac{1}{2} \notin f_{\delta}^{M}\left(\bar{E}_{\delta}\right)$. 
(2) In this case $\frac{1}{2} \in f_{\delta}^{M}\left(\bar{E}_{\delta}\right)$ and we have $N=M$ since

$$
\bar{E}_{\delta} \cap f_{\delta}^{n}\left(\bar{E}_{\delta}\right)=\emptyset \text { if } n<M .
$$

Proposition 7.6. If $\delta \in \mathbf{J}\left(\delta_{0}\right)$, then $f_{\delta}$ has no restrictive central point.

Proof. From the definition of $\mathbf{J}\left(\delta_{0}\right)$ we only have to focus our attention on those restrictive central points of period less than $\delta^{-1 / 2}$. We first show that there are no restrictive central points in $E_{\delta}$. Let $x$ be such a restrictive central point of period $n$ and let $K=\{x, 1-x\}_{ \pm}$. We have $f_{\delta}^{n}(K) \subset K$ and since $\frac{1}{2} \in K, \frac{1}{2} \in f_{\delta}^{r n}(K)$ for any $r$ in $\mathbb{N}$. However, $K \subset E_{\delta}$ implies a contradiction with $\delta \notin \mathbf{I}_{n}^{5}$.

We now concentrate on those restrictive central points which are outside $E_{\delta}$, and we first show that their period is less than or equal to $N$. Let $x \notin E_{\delta}$ be periodic with period $n>N$. Assume first $x<\frac{1}{2}$. By Lemma 7.5 there is a $y$ such that $x<y<\frac{1}{2}$ and $f_{\delta}^{N}(y)=\frac{1}{2}$. This implies $f_{\delta}^{n^{\prime}}(y)=0$. However since $S(f) \leqq 0$ if $x \neq \frac{1}{2}-\delta, \frac{1}{2}+\delta$, $f_{\delta}^{n^{\prime}}(y)=0$ implies that $y$ is a local minimum or a local maximum for $f_{\delta}^{n}$, [4]. This implies that $f_{\delta}^{n}$ is not a homeomorphism on $\left[x, \frac{1}{2}\right]$, therefore $x$ cannot be central. The case $x>\frac{1}{2}$ is similar.

We shall now exclude the remaining possibility, namely a restrictive central point of period less than or equal to $N, p_{n} \notin E_{\delta}$. We have $f_{\delta}\left(\frac{1}{2}\right)=1-\delta, f_{\delta}^{2}\left(\frac{1}{2}\right)=2 \delta$, $f_{\delta}^{n+1}\left(\frac{1}{2}\right)=2^{n} \delta$ as long as $f_{\delta}^{n}\left(\frac{1}{2}\right) \notin E_{\delta}$. We now compute the position of the fixed point $p_{n}>\frac{1}{2}$ of $f_{\delta}^{n}$ which is the nearest to $\frac{1}{2}$ for $n \leqq N$. Notice that this is the only candidate for a restrictive central point of period $n \leqq N$. Since $p_{n} \notin E_{\delta}, f_{\delta}$ can be replaced by the broken linear transformation defined by

$$
g(x)=\left\{\begin{array}{lll}
2 x & \text { if } & 0 \leqq x \leqq \frac{1}{2} \\
2(1-x) & \text { if } & \frac{1}{2} \leqq x \leqq 1
\end{array}\right.
$$

Let $q_{n}$ be the fixed point of $g^{n}$ which is the nearest to $\frac{1}{2}$ and greater than $\frac{1}{2}$. By a direct computation, one obtains $q_{n}=\frac{1}{2}\left(1-2^{-n}\right)^{-1}$. If $2<n<M$, we have

$$
\frac{1}{2}-f_{\delta}^{n}\left(\frac{1}{2}\right)=\frac{1}{2}-2^{n-1} \delta>\frac{2^{-n-1}}{1+2^{-n}}=q_{n}-\frac{1}{2} .
$$

Thus $p_{n}$ cannot be central and restrictive in this case, cf. Fig. 2.

It is easy to exclude directly the occurrence of a restrictive central point of period one or two. The only remaining possible periods for a restrictive central point are $M$ and $M+1$ if $N=M+1$, and $M$ if $N=M$. We investigate the two cases separately.

Case 1. $N=M+1$. This case corresponds to Case 1 of Lemma 7.5. We have $f_{\delta}^{M}\left(\frac{1}{2}\right)$ $=2^{M-1} \delta<\frac{1}{2}$, and $f_{\delta}^{M+1}\left(\frac{1}{2}\right)=2^{M} \delta<\frac{1}{2}$. Since $f_{\delta} \leqq g$ and $f_{\delta}^{M}\left(\frac{1}{2}-\delta\right)=2^{M} \delta<\frac{1}{2}$, we have $f_{\delta}^{M}(x)>g^{M}(x)$ for $x \in E_{\delta}$. From this we have $\frac{1}{2}<p_{M}<q_{M}$ and therefore $p_{M} \in E_{\delta}$. But we have seen that such points are not central restrictive if $\delta \in \mathbf{J}\left(\delta_{0}\right)$. However no other fixed point of $f_{\delta}^{M}$ can be central, and so $f_{\delta}^{M}$ has no restrictive central fixed point. We now investigate $f_{\delta}^{M+1}$. We have $f_{\delta}^{M+1}\left(p_{M}\right)=f_{\delta}\left(p_{M}\right) \geqq 1-2 \delta>p_{M}$ since $p_{M}$ belongs to $E_{\delta}$. Moreover $f_{\delta}^{M+1}\left(\frac{1}{2}\right)<\frac{1}{2}$, therefore by continuity there is a solution $y$ of $f_{\delta}^{M+1}(x)=x$ such that $p_{M}>x>\frac{1}{2}$. This implies $p_{M+1} \in E_{\delta}$, and as before $p_{M+1}$ cannot be central and restrictive. 


\section{Fig. 2}

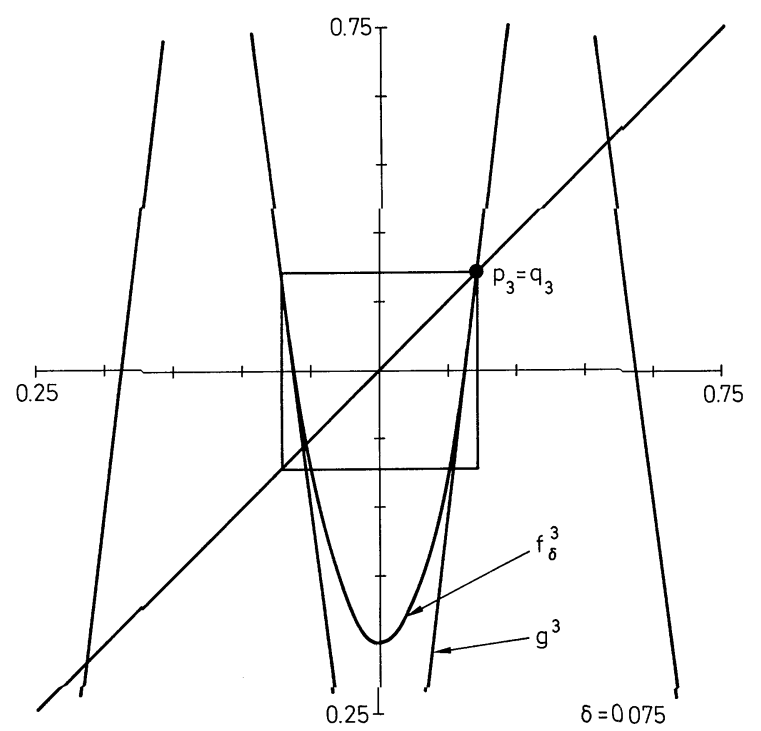

Case 2. $N=M$. This case corresponds to Case 2 of Lemma 7.5. We have $f_{\delta}^{M}\left(\frac{1}{2}\right)$ $=2^{M-1} \delta \notin E_{\delta}$. Consider the equation for $v$,

$$
2^{M-1} \delta\left(1+v^{2}\right)=\frac{1}{2} \text {. }
$$

This equation has a solution $v$ with $0<v<1$ since

$$
(1-2 \delta)^{-1} \leqq 2^{-M} \delta^{-1} \leqq 2 .
$$

For this $v$ we have

$$
f_{\delta}^{M-1}\left(\frac{1}{2}+v \delta\right)=2^{M-2} \delta\left(1+v^{2}\right)=\frac{1}{4} \notin E_{\delta} .
$$

Therefore

$$
f_{\delta}^{M}\left(\frac{1}{2}+v \delta\right)=2^{M-1} \delta\left(1+v^{2}\right)=\frac{1}{2} .
$$

From this and the monotonicity of $f_{\delta}^{M}$ on $\left[\frac{1}{2}, \frac{1}{2}+v \delta\right]$, we deduce

$$
p_{M} \in\left[\frac{1}{2}, \frac{1}{2}+v \delta\right] \subset E_{\delta} .
$$

As before, this implies that $p_{M}$ is not central and restrictive.

Proposition 7.7. $\bigcup_{m=1}^{\infty} \mathbf{I}_{m}^{5}$ has Lebesgue measure zero.

Proof. This will be an immediate consequence of the fact that every $\mathbf{I}_{m}^{5}$ has Lebesgue measure zero. There are three cases.

Case 1. $2^{-q-1} \leqq \delta<\inf \left(\delta_{0}, 2^{-q}\right)$ and $m<q-3, q \leqq q_{0}$. In this case Lemma 1.1 implies that $\mathbf{I}_{m}^{5}=\emptyset$. 
Case 2. $2^{-q-1} \leqq \delta<\inf \left(\delta_{0}, 2^{-q}\right), q-3 \leqq m<2^{q / 2}, q \leqq q_{0}$. We shall prove that $\lambda\left(\mathbf{I}_{m, q, k}^{5}\right) \leqq \mathcal{O}(1) 2^{-k q / 8} 2$. If $\delta \in \mathbf{I}_{m, q, k}^{5}$, we define the numbers $l_{1}, l_{2}, \ldots, l_{k}$ by

$$
\sum_{i=1}^{l_{j}} n_{i}=m \cdot j \quad \text { for } j=1,2, \ldots, k .
$$

From this we deduce that every $n_{i}$ is in this case less than $\delta^{-1 / 2}$. The bound on $Z$ in Lemma A. 3 is now

$$
\begin{aligned}
Z \leqq & \left(2^{\alpha} q^{\alpha}(\log 2)^{\alpha}+2\right) 2^{-q(1-\alpha / 2)}\left(1-2^{(\alpha / 2-1)}\right)^{-1} \\
& +\mathcal{O}(1) \sum_{n=q-4}^{2(q+1) / 2} 2^{-q-n \frac{2-\alpha}{2} 2^{-(q+1)}} \\
\leqq & 2^{-q / 5},
\end{aligned}
$$

if $\alpha \leqq \frac{3}{2}$. Let $W_{s, q}^{5}$ be the volume of the set of those $\delta$ in $\mathbf{J}_{\infty}$, for which $2^{-q-1} \leqq \delta$ $<2^{-q}$, and $n_{1}, n_{2}, \ldots, n_{s}<2^{(q+1) / 2}$, and $v_{s} \in(-1,1)$. We use again Corollary 5.2 and bound $R\left(Q_{s-1}\right)$. We have $R\left(Q_{s-1}\right) \geqq \Delta^{-5 s}$ from Lemma 6.4. We now bound $W_{s, q}^{5}$ as in the case of $V_{s, q}^{2}$. We obtain

$$
\begin{aligned}
W_{s, q}^{5} \leqq & \sum_{l \geqq 0} \sum_{0<p_{0}<\ldots<p_{l}<s} \sum_{B_{1}, n_{1}}^{q} 2^{-n_{1}} \delta^{\left|\Sigma_{s-1}\right| / 5} \\
& \cdot \sum_{p_{j}<t_{j} \leqq p_{j+1}, j<l} 8 \delta \prod_{j=0}^{l}\left(t_{j}-p_{j}\right)^{-2} 2^{-n_{1}\left|T_{s-1} \cup T_{s-1}^{\prime}\right| / 8} \\
\leqq & (1) \delta^{s / 8} .
\end{aligned}
$$

Since $\lambda\left(\mathbf{L}_{m, k}^{5}\right) \leqq \sum_{q=q_{0}}^{\infty} W_{m k, q}^{5}$, we have

$$
\lambda\left(\mathbf{L}_{m, k}^{5}\right) \leqq \mathcal{O}(1) 2^{-m k q_{0} / 10} \leqq \mathcal{O}(1) 2^{-k}
$$

if $q_{0}=\left[\log _{2} \delta_{0}^{-1}\right]$ is sufficiently large. From $\mathbf{I}_{m}^{5}=\bigcap_{k} \mathbf{L}_{m, k}^{5}$, we have $\lambda\left(\mathbf{I}_{m}^{5}\right)=0$.

Case 3. $2^{-q-1} \leqq \delta<\inf \left(\delta_{0}, 2^{-q}\right)$ and $m>2^{q / 2}\left(q \leqq q_{0}\right)$. Let

$$
E_{\delta / \Delta}=\left(\frac{1}{2}-\delta / \Delta, \frac{1}{2}+\delta / \Delta\right) \text {. }
$$

We shall prove first that $\frac{1}{2} \in f_{\delta}^{n}\left(E_{\delta / \Delta}\right)$ for some $n<3 \Delta$. As long as $f_{\delta}^{n}\left(E_{\delta / \Delta}\right) \cap E_{\delta}=\emptyset$, we have

$$
f_{\delta}^{n}\left(E_{\delta / \Delta}\right)=\left\{2 A_{n}+\sigma_{n} 2^{n} \delta, 2 A_{n}+\sigma_{n} 2^{n}\left(1+\Delta^{-2}\right) \delta\right\}_{ \pm},
$$

where $A_{n} \in \mathbb{Z}$ and $\sigma_{n}= \pm 1$. We define $a_{n}$ and $b_{n}$ by $f_{\delta}^{n}\left(E_{\delta / \Delta}\right)=\left(a_{n}, b_{n}\right)$. We notice that $\left|a_{n}-b_{n}\right|=2^{n} \delta \Delta^{-2}$ which implies $f_{\delta}^{M_{1}}\left(E_{\delta / \Delta}\right) \cap E_{\delta} \neq \emptyset$ for some smallest $M_{1}<2 \Delta$. There are three cases.

Case 3.1. $\frac{1}{2} \in f_{\delta}^{M_{1}}\left(E_{\delta / \Delta}\right)$, then the assertion is proven.

Case 3.2. $\frac{1}{2} \notin f_{\delta}^{M_{1}}\left(E_{\delta / \Delta}\right)$ and $a_{M_{1}} \in E_{\delta}$ (notice that from $M_{1}>\Delta-3$, we have $\left.\left|b_{M_{1}}-a_{M_{1}}\right| \geqq \mathcal{O}(1) \Delta^{-2} \gg 2 \delta\right)$. This implies

$$
E^{1} \equiv\left[\frac{1}{2}+\delta, \frac{1}{2}+\Delta^{-3}\right] \subset f^{M_{1}}\left(E_{\delta / \Delta}\right) \text {. }
$$

$2 \lambda$ denotes Lebesgue measure 
However, if $j>1$, we have

$$
f_{\delta}^{j}\left(E^{1}\right)=\left[2^{j} \delta, 2^{j} \Delta^{-3}\right],
$$

as long as $2^{j-1} \Delta^{-3}<\frac{1}{2}-\delta$. Let $j_{0}$ be the smallest integer such that $2^{j_{0}} \Delta^{-3} \geqq \frac{1}{4}$. We have $\frac{1}{4} \in f_{\delta}^{j_{0}}\left(E^{1}\right)$, since from $2^{j_{0}-1} \Delta^{-3}<\frac{1}{4}$ we deduce $\delta 2^{j_{0}}<\frac{1}{8}$. Therefore $\frac{1}{2} \in f_{\delta}^{j_{0}+1}\left(E^{1}\right) \subset f_{\delta}^{j_{0}+M_{1}+1}\left(E_{\delta / 4}\right)$. The assertion is proven in this case since $1+j_{0}+M_{1}$ $<3 \Delta$.

Case 3.3. $\frac{1}{2} \notin f_{\delta}^{M_{1}}\left(E_{\delta / \Delta}\right)$ and $b_{M_{1}} \in E_{\delta / \Delta}$. This implies

$$
E^{2} \equiv\left[\frac{1}{2}-\Delta^{-3}, \frac{1}{2}-\delta\right] \subset f_{\delta}^{M_{1}}\left(E_{\delta / \Delta}\right) .
$$

However, if $j>1$, we have

$$
f_{\delta}^{j}\left(E^{2}\right)=\left[2^{j} \delta, 2^{j} \Delta^{-3}\right],
$$

as long as $2^{j-1} \Delta^{-3}<\frac{1}{2}-\delta$. This implies as in Case 3.2 that $\frac{1}{2} \in f_{\delta}^{M_{1}+j_{0}+1}\left(E_{\delta / \Delta}\right)$ for some $j_{0}$ such that $1+j_{0}+M_{1}<3 \Delta$. Assume now there is a restrictive central point $p$, of period greater than $5 \Delta$ and such that $p \notin E_{\delta / \Delta}$. From the preceding argument, there is a $y \in\left\{\frac{1}{2}, p\right\}_{ \pm}$such that $f^{n}(y)=\frac{1}{2}$ for some $n<4 \Delta-1$. This implies $f_{\delta}^{j^{\prime}}(y)=0$ if $j>4 \Delta$. Let $q$ be the period of $p$, since $S(f) \leqq 0$ if $x \neq \frac{1}{2}-\delta, \frac{1}{2}+\delta, f_{\delta}^{q^{\prime}}(y)=0$ implies that $y$ is a local maximum or a local minimum of $f^{q}$. This is a contradiction with the fact that $p$ is central.

We now recapitulate the possibilities for central restrictive points.

(1) There is no central restrictive point outside $E_{\delta}$ (Proposition 7.4).

(2) In $E_{\delta} \backslash E_{\delta / \Delta}$, every central restrictive point is of period less than $5 \Delta$.

However the values of $\delta$ for which a central restrictive point occurs in $E_{\delta} \backslash E_{\delta / \Delta}$ belong to $\bigcup_{m=1}^{5 q} \mathbf{I}_{m, q}^{5}$ which is of measure zero (Case 2). Therefore, we only have to estimate the volume of $\mathbf{I}_{m, q}^{5}$ for $m>2^{-q / 2}$, and in the case where the restrictive central point is in $E_{\delta / \Delta}$. Let $p$ be such a restrictive central point for some $\delta \in \mathbf{I}_{m}^{5}$. Let $K=\{p, 1-p\}_{ \pm}$, we have $f_{\delta}^{m}(K) \subset K \subset E_{\delta / \Delta}$ and since $\frac{1}{2} \in K, f_{\delta}^{r m}\left(\frac{1}{2}\right) \in E_{\delta}$ for any $r \geqq 1$. This implies $\left|v_{r m}\right|<\Delta^{-1}$. From this and

$$
v_{r m}^{2}\left(B, v_{r m+1}\right) \geqq \mathcal{O}(1)\left|B-B_{c}\right| /\left(\delta 2^{n_{r m+1}}\right)
$$

if $B \neq B^{c}$ (of Lemma A.3) we deduce

$$
|B| \leqq \delta \Delta^{-1} 2^{n_{r m+1}} \text {. }
$$

The conclusion of Lemma A.3 is therefore replaced by

$$
Z \leqq \mathcal{O}(1) \Delta^{-1} \leqq q^{-1 / 2} \text {. }
$$

Let $\mathbf{M}_{m, k, q}^{5}$ be the set of those $\delta, \delta \in \mathbf{J}_{\infty}, 2^{-q-1} \leqq \delta<\inf \left(2^{-q}, \delta_{0}\right), q>q_{0}$, and such that $\left|v_{r m}\right| \leqq \Delta^{-1}$ for $r=1,2, \ldots, k$. Let $\mathbf{M}_{m, k}^{5}=\bigcup_{q=q_{0}}^{\infty} \mathbf{M}_{m, k, q}^{5}$, we shall now prove the following bound

$$
\lambda\left(\mathbf{M}_{m, k}^{5}\right) \leqq \mathcal{O}(1) 2^{-k} .
$$

This will imply $\lambda\left(\mathbf{I}_{m}^{5}\right)=0$ since $\mathbf{I}_{m}^{5} \subset \bigcap_{k=1}^{\infty} \mathbf{M}_{m, k}^{5}$ by the preceding argument. Let $V_{m, k, q}^{5}$ be the Lebesgue measure of $\mathbf{M}_{m, k, q}^{5} \cdot v_{k m+1}$ is any number between -1 and +1 . The 
bound for $V_{m, k, q}^{5}$ is the usual one. However, in the summation we have to precise those $r$ for which $r_{i} \in \mathbb{P}$. We obtain

$$
\begin{aligned}
& V_{m, k, q}^{5} \leqq \sum_{i=0}^{k} 2^{-q i / 2} \sum_{\substack{0<p_{1}<\ldots<p_{l} \leqq k m \\
r_{1}<\ldots<r_{k}-i}} \sum_{B_{1}, n_{1}}^{q} 2^{-n_{1}} \\
& \cdot \sum_{p_{j}<t_{j} \leqq p_{j+1}, j<l} \mathcal{O}(1) \delta \prod_{j=0}^{l}\left(t_{j}-p_{j}\right)^{-2} \\
& \cdot 2^{-n_{1}\left|T_{k m-1} \cup T_{k m-1}^{\prime}\right| / 16} q^{-r(k m) / 16} \Delta^{-2\left|\Sigma_{k m-1}\right|} \\
& \leqq \mathcal{O}(1) k q^{-k / 20} \leqq q^{-k / 30} \text {. }
\end{aligned}
$$

Since $\lambda\left(\mathbf{M}_{m, k}^{5}\right) \leqq \sum_{q=q_{0}}^{\infty} V_{m, k, q}^{5}$, we have, for $k>40$,

$$
\lambda\left(\mathbf{M}_{m, k}^{5}\right) \leqq q_{0}^{-k / 40} \leqq 2^{-k},
$$

which ends the proof of the proposition.

Combining Proposition 7.5, Theorems 7.1-7.3 we obtain Theorems A and B.

\section{Appendix}

Lemma A.1. Let $f(n, q)$ be the number of sequences of binary numbers with $n$ digits but not more than $q-1$ consecutive zeros. Then

$$
f(n, q) \leqq 3 \cdot 2^{n} \exp \left(-n / 2^{q+1}\right),
$$

for $q>1$.

Proof. The following recursive relations hold for $n>q$,

$$
f(n+1, q)=2 f(n, q)-f(n-q, q) .
$$

This can be seen by appending a zero or a one to every allowed sequence with $n$ digits and subtracting the number of sequences which end as $1 \underbrace{0 \ldots 0}_{q}$, i.e. for which only $n-q$ digits are arbitrary. No solution to (A.2) can grow faster than $A(q) x_{0}^{n}$, where $x_{0}$ is the solution of largest modulus of $x^{q+1}=2 x^{q}-1$. We find, for large $q, x_{0} \sim 2-2^{-q}$ or $x_{0} \leqq 2 \exp \left(-\left(\frac{1}{2}\right)^{q+1}\right)$. In order to derive a bound on $A(q)$ which is uniform in $q$ it is more convenient to consider

$$
g(n, q)=f(n, q)-f(n-1, q) .
$$

Since $f(j, q)=2^{j}$ for $j=1, \ldots, q-1$ and $f(q, q)=2^{q}-1$,

$$
f(q+1, q)=2^{q+1}-3
$$

we find

$$
g(n, q) \leqq 2^{n} \exp \left(-n / 2^{q+1}\right),
$$

for $n \leqq q+1$. From (A.2), we have

$$
g(n+1, q)=\sum_{j=n-q+1}^{n} g(j, q)
$$


and so we should verify for $n>q$,

or

$$
2^{n+1} \exp \left(-(n+1) / 2^{q+1}\right) \geqq \sum_{j=n-q+1}^{n} 2^{j} \exp \left(-j / 2^{q+1}\right)
$$

$$
1 \geqq\left(1-\left(2 \exp \left(-2^{-(q+1)}\right)\right)^{-q}\right) /\left(2 \exp \left(-2^{-(q+1)}\right)-1\right) .
$$

But this is true and thus (A.3) follows in all cases. Since $f(n, q)=\sum_{j=2}^{n} g(j, q)+2$, the bound (A.1) follows by inspection.

Lemma A.2. Let $g\left(n, q, B_{c}\right), B_{c}>0$ be the number of sequences of binary numbers $B$ with not more than $q-1$ consecutive zeros, satisfying

$$
2^{n-1} \leqq\left|B-B_{c}\right|<2^{n} \text {. }
$$

Then $g(n, q) \leqq \mathcal{O}(1) 2^{n} \exp \left(-n / 2^{q+5}\right)$ for $q>5$.

Proof. We reduce this lemma to the preceding one. Since $B_{c}>0$, we can write $B_{c}=2^{n-2} a+b$ with $a, b \in \mathbb{N}, b<2^{n-2}$. If $B>B_{c}$, then we must have

$$
2^{n-2} a+b+2^{n-1}<B<2^{n-2} a+b+2^{n},
$$

which is satisfied if

$$
2^{n-2} a+2^{n-2}<B<2^{n-2} a+2^{n+1} .
$$

Writing $B=2^{n-2} a+r$, we are asking thus how many $r$ there are such that

$$
2^{n-2}<r<2^{n+1}
$$

with not more than $q+4$ consecutive zeros, (because the highest order digits of $r$ may be influenced through $2^{n-2} a$ ). But this is bounded through Lemma A.1, by

$$
3 \cdot 3 \cdot 2^{n} \exp \left(-(n-2) / 2^{q+5}\right) \text {. }
$$

Substituting the result of Corollary 5.2 into the bounds for $V_{s, q}^{1}$, we see that it is useful to have a general bound, for all $j \in \Sigma_{s-1}$ (in particular $j \notin \mathbb{P}$ ), of the quantity

$$
\sum_{B_{j+1}, n_{j+1}}^{q} \sup _{\delta} *\left|v_{j}\right|^{-1} 2^{-n_{j+1}}
$$

To be more specific, we extend the sum and the sup somewhat by introducing the following set for which the bound will be proven. Let

$$
X_{q}=\left\{n, B \mid n \geqq q-4 \text {, and } B \text { compatible with } n \text { and some } \delta \in \mathbf{K}_{q}\right\} \text {. }
$$

Lemma A.3. For some universal constant $C$, we have, for $1 \leqq \alpha \leqq 3 / 2$,

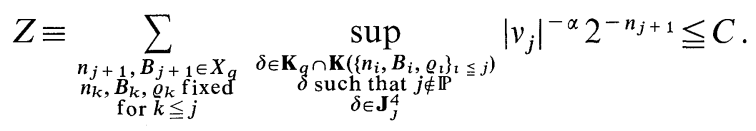

Proof. By the equality

$$
\delta=\frac{B_{j+1}}{2^{n_{j+1}-1}\left(1+v_{j}^{2}\right)+v_{j+1}}=\frac{B_{1}}{2^{n_{1}-1}+v_{1}},
$$


we find

$$
v_{j}^{2}=\left(\frac{B_{j+1} 2^{n_{1}}}{B_{1} 2^{n_{j+1}}}\left(1+\frac{v_{1}}{2^{n_{1}-1}}\right)-1-\frac{v_{j+1}}{2^{n_{j+1}-1}}\right) .
$$

Since, by construction, $j \notin \mathbb{P}$, we find by Lemma 2.3 that we have to sum at most over the set $v_{j}^{2} \geqq 2^{-n_{j+1}} \Delta^{-4}$. Therefore, we have to sum at most over the set (with $\left.B=B_{j+1}, n=n_{j+1}\right)$,

$$
\left\{n, B \mid n \geqq q-5,1 \geqq v_{j}^{2} \geqq 2^{-n} \Delta^{-4} \equiv \varrho^{2}\right\} .
$$

We consider, for fixed $n_{1}, B_{1}, \ldots, n_{j+1}$, the function $v_{j}^{2}$ as a function of $B=B_{j+1}$ and $v_{j+1}$. In order to simplify the expressions, we consider $B$ as a continuous variable, but the correct argument must use difference calculus instead of differential calculus. Then

$$
\frac{\partial v_{j}^{2}}{\partial B}=\frac{2^{n_{1}}}{B_{1} 2^{n_{j+1}}}\left(1+\frac{v_{1}\left(v_{j}\right)}{2^{n_{1}-1}}\right) \cdot\left(1-\frac{B}{2 B_{1} 2^{n_{j+1}} v_{j}} \frac{\partial v_{1}}{\partial v_{j}}\right)^{-1}
$$

and

$$
\frac{\partial v_{j}^{2}}{\partial v_{j+1}}=-\frac{1}{2^{n_{j+1}-1}}\left[1-\frac{B}{2 B_{1} 2^{n_{j+1}} v_{j}} \frac{\partial v_{1}}{\partial v_{j}}\right]^{-1} .
$$

By the previous exclusions, and the method of p. 142, we find

$$
\frac{2}{3} \leqq\left[1-\frac{B}{2 B_{1} 2^{n_{j+1}} v_{j}} \frac{\partial v_{1}}{\partial v_{j}}\right]^{-1} \leqq \frac{3}{2} .
$$

Therefore

$$
\left|\frac{\partial v_{j}^{2}}{\partial B}\right| \geqq \frac{\mathcal{O}(1)}{2^{n_{j+1}} \delta} \quad \text { and } \quad\left|\frac{\partial v_{j}^{2}}{\partial v_{j+1}}\right| \leqq \frac{\mathcal{O}(1)}{2^{n_{j+1}}} .
$$

Suppose now that for some value $B^{c}$ of $B$ and $v^{c}$ of $v_{j+1}$, we find

$$
\left|v_{j}\right|<2^{-n_{j+1} / 2} \text {. }
$$

Then for every $B \neq B^{c}$, the above bounds imply by integration

$$
\begin{aligned}
v_{j}^{2}\left(B, v_{j+1}\right) \geqq & \mathcal{O}(1) \frac{\left|B-B^{c}\right|}{\delta 2^{n_{j+1}}}-\frac{\left|v_{j+1}-v^{c}\right|}{2^{n_{j+1}}} \\
& -v_{j}^{2}\left(B^{c}, v^{c}\right) \\
\geqq & \mathcal{O}(1)\left|B-B^{c}\right| /\left(\delta 2^{n_{j+1}}\right),
\end{aligned}
$$

since $\left|B-B^{c}\right| \geqq 1$. In all these expressions, $\delta$ stands for its worst possible value. The bound on $Z$ is therefore

$$
\begin{aligned}
Z \leqq & \sum_{n \geqq q-4} 2^{-n} \varrho^{-\alpha} \\
& +\sum_{\substack{n, B \in X^{q} \\
B \neq B^{c}}} 2^{-n}\left[\frac{\left|B-B^{c}\right|}{2^{n}} u\right]^{-\alpha / 2},
\end{aligned}
$$


where $u=\mathcal{O}\left(\delta^{-1}\right)$. For fixed $n$, we split the second sum over $B$ into two parts corresponding to

$$
\tau^{2} \geqq 2^{-n}\left|B-B^{c}\right| u \geqq 2^{-n+2}
$$

and $1 \geqq \frac{\left|B-B^{c}\right|}{2^{n}} u \geqq \tau^{2}$, where $\tau^{2}$ will be fixed below. The first part is bounded by

$$
\begin{aligned}
& 2^{-n+1-\alpha(2-n) / 2}+2 \underset{\tau^{2} \geqq 2-n_{u z} \geqq 2^{2-n}}{ } d z 2^{-n}\left[2^{-n} u z\right]^{-\alpha / 2} \\
& \leqq 2^{(\alpha / 2-1) n+1}+\frac{2}{1-\alpha / 2} \cdot u^{-1} \tau^{2-\alpha} .
\end{aligned}
$$

For the second term we use the bound $2^{-2} \tau^{-\alpha}$ times the number of terms. The number of choices of $B$, compatible with $n$, and some $\delta \in \mathbf{K}_{q}$, according to Lemma A.1, taking $f(n-q, q)$, is bounded by

$$
3 \cdot 2^{n-q} \exp \left(-(n-q) / 2^{q+1}\right) \leqq 4 \cdot 2^{n-q-n / 2^{q+1}} .
$$

We thus get the final bound

$$
Z \leqq \sum_{n \geqq q-4}\left[2^{-n} \varrho^{-\alpha}+2^{-n(1-\alpha / 2)+1}+\frac{2}{1-\alpha / 2} \cdot u^{-1} \tau^{2-\alpha}+4 \cdot 2^{-q-n / 2^{q+1}} \tau^{-\alpha}\right] .
$$

Optimizing with respect to $\tau$ and using $u=\mathcal{O}\left(2^{q}\right)$ we get

$$
\begin{aligned}
Z \leqq & \left(2^{\alpha} q^{\alpha}(\log 2)^{\alpha}+2\right) 2^{-q(1-\alpha / 2)}\left(1-2^{(\alpha / 2-1)}\right)^{-1} \\
& +\sum_{n \geqq q-4} \mathcal{O}(1) 2^{-q} 2^{-n \frac{2-\alpha}{4} 2^{-q}} \leqq C .
\end{aligned}
$$

This completes the proof of the lemma.

Another typical sum in the final estimates is the one for which $j=t_{l}=t\left(p_{l}\right)$, when $t_{l}<p_{l+1}$, i.e. when $j \in T_{s}$. The expression obtained through Corollary 5.2 is then, with fixed $p\left(=p_{l}\right)$.

$$
Z^{\prime} \equiv \sum_{\substack{n_{j+1}, B_{j+1} \in X_{q} \\ n_{k}, B_{k}, e_{k}, k \leqq j \\ \text { fixed }}} \sup _{\substack{\delta \in \mathbf{K}_{q} \cap \mathbf{K}\left(\left\{n_{i}, B_{i}, e_{i}\right\}_{i} \leq j\right) \\ \delta \text { such that } j=t(p)<\bar{f}(p) \\ \delta \in \mathbf{J}_{j}^{4}}}\left[2^{n_{j+1}}\left|v_{j}\right|\left|v_{j}-v_{j-p}\right|^{1 / 2}\right]^{-1} .
$$

\section{Lemma A.4}

$$
Z^{\prime} \leqq C \Delta^{10(j-p+1)} \text {. }
$$

Proof. We first give a general bound on $2^{n_{j+1}}\left|v_{j}\right|\left|v_{j}-v_{j-p}\right|^{1 / 2}$. We distinguish three cases.

Case 1. $\left|v_{j-p}\right| \geqq 2\left|v_{j}\right|$. Then

$$
\left|v_{j-p}-v_{j}\right| \geqq\left|v_{j-p}\right| / 2 \geqq\left|v_{j}\right| \text {. }
$$

Therefore

$$
2^{-n_{j+1}}\left|v_{j}\right|^{-1}\left|v_{j}-v_{j-p}\right|^{-1 / 2} \leqq 2^{-n_{J}+1}\left|v_{j}\right|^{-3 / 2} .
$$


Case 2. $\left|v_{j-p}\right|<2\left|v_{j}\right|$ and $v_{j} v_{j-p}>0$. Then

$$
\begin{aligned}
2^{-n_{J+1} \mid}\left|v_{j}\right|^{-1}\left|v_{j}-v_{j-p}\right|^{-1 / 2} & =2^{-n_{j+1}}\left|v_{j}\right|^{-1} \frac{\left(\left|v_{j}\right|+\left|v_{j-p}\right|\right)^{1 / 2}}{\left|v_{j}^{2}-v_{j-p}^{2}\right|^{1 / 2}} \\
& \leqq 3 \cdot 2^{-n_{j+1}}\left|v_{j}\right|^{-1 / 2}\left|v_{j}^{2}-v_{j-p}^{2}\right|^{-1 / 2} \\
& \leqq 6 \cdot 2^{-n_{j+1}}\left|v_{j-p}\right|^{-1 / 2}\left|v_{j}^{2}-v_{j-p}^{2}\right|^{-1 / 2} \\
& \leqq 6 \cdot 2^{-n_{j+1}} \Delta^{2(j-p)}\left|v_{j}^{2}-v_{j-p}^{2}\right|^{-1 / 2}
\end{aligned}
$$

by Lemma 2.5 .

Case 3. $\left|v_{j-p}\right|<2\left|v_{j}\right|$ and $v_{j} v_{j-p}<0$. Then

$$
2^{-n_{j+1}}\left|v_{j}\right|^{-1}\left|v_{j}-v_{j-p}\right|^{-1 / 2} \leqq 2^{-n_{j}+1}\left|v_{j}\right|^{-3 / 2} \text {. }
$$

Combining the three cases, we obtain

$$
2^{-n_{j+1}}\left|v_{j}\right|^{-1}\left|v_{j}-v_{j-p}\right|^{-1 / 2} \leqq 2^{-n_{j+1}}\left|v_{j}\right|^{-3 / 2}+6 \cdot 2^{-n_{j+1}} \Delta^{2(j-p)}\left|v_{j}^{2}-v_{j-p}^{2}\right|^{-1 / 2} .
$$

For the term $2^{-n_{3}+1}\left|v_{j}\right|^{-3 / 2}$, the bound on the sum follows by Lemma A.3. We now give the estimate for the other term. First of all, we fix $n_{j-p+1}$ and $B_{j-p+1}$, and set $n=n_{j+1}$ and $B=B_{j+1}$. We have

$$
\begin{aligned}
\left|v_{j}^{2}-v_{j-p}^{2}\right|= & \frac{B 2^{n_{1}}}{B_{1} 2^{n}}\left(1+\frac{v_{1}}{2^{n_{1}-1}}\right) \\
& -\frac{B_{j-p+1}}{2^{n_{j-p+1}} B_{1}}\left(1+\frac{v_{1}}{2^{n_{1}-1}}\right)+\frac{v_{j-p+1}}{2^{n_{j-p+1}-1}}-\frac{v_{j+1}}{2^{n-1}} .
\end{aligned}
$$

We distinguish two cases.

Case 1. $n \leqq n_{j-p+1}$. In this case, there is at most one value of $B$ (call it $B_{c}^{\prime}$ ) such that

$$
\left|v_{j}^{2}-v_{j-p}^{2}\right|<2^{-n-1} \delta^{-1} \text {. }
$$

This is due to the fact that

$$
\begin{aligned}
\frac{\partial v_{1}}{\partial B} & =\frac{\partial v_{1} \partial v_{j}}{\partial v_{j} \partial B}=\frac{1}{2 v_{j}} \frac{\partial v_{1}}{\partial v_{j}} \frac{\partial v_{j}^{2}}{\partial B} \\
& =\frac{2^{n_{1}-1}}{B_{1} 2^{n^{n} v_{j}}}\left(1+\frac{v_{1}}{2^{n_{1}-1}}\right)\left(1-\frac{B}{B_{1} 2^{n_{j+1}+1} v_{j}} \frac{\partial v_{1}}{\partial v_{j}}\right)^{-1} \frac{\partial v_{1}}{\partial v_{j}},
\end{aligned}
$$

where we have considered $B$ as a continuous variable, as in the estimate of $Z$. Therefore, since $j \notin \mathbb{P}$,

$$
\begin{aligned}
\left|\frac{\partial v_{1}}{\partial B}\right| & \leqq \mathcal{O}(1) \delta^{-1} \Delta^{2} 2^{-n / 2} R_{j-1}^{-1} \\
& \leqq \mathcal{O}(1) \delta^{-1 / 2} \Delta^{2} \delta^{1 / 8+(j-1) / 10} \leqq \Delta^{2} \delta^{-1 / 2}
\end{aligned}
$$

as in Proposition 6.2. From this we deduce

$$
\left|\frac{\partial}{\partial B}\left[\frac{v_{1}}{2^{n_{1}}} \frac{B_{j-p+1} 2^{n_{1}}}{2^{n_{j}-p+1} B_{1}}\right]\right|=\frac{B_{j-p+1} 2^{n_{1}}}{2^{n_{j-p+1}} B_{1}} \cdot 2^{-n_{1}}\left|\frac{\partial v_{1}}{\partial B}\right| \leqq \delta^{1 / 3} .
$$


Therefore if $B \neq B_{c}^{\prime}$, we have

$$
\begin{aligned}
\left|v_{j}^{2}-v_{j-p}^{2}\right| & =\left|B 2^{-n} \delta^{-1}-B_{j-p+1} 2^{-n_{j-p+1}} \delta^{-1}+\mathcal{O}\left(2^{-n}\right)\right| \\
& =2^{-n} \delta^{-1}\left|B-2^{-l} B_{j-p+1}+\mathcal{O}(\delta)\right| \\
& \geqq 2^{-n-1} \delta^{-1}\left|B-2^{-l} B_{j-p+1}\right| \geqq 2^{-n-1} \delta^{-1},
\end{aligned}
$$

where $l=n_{j-p+1}-n$. From Lemma 2.6 we have (even if $B=B^{c}$ ),

$$
\left|v_{j}^{2}-v_{j-p}^{2}\right|^{1 / 2} \geqq 2^{-n / 2} \Delta^{-8(j-p)-2} .
$$

With $u=\delta^{-1} / 2, B_{c}=2^{-l} B_{j-p+1}, \alpha=1$, the summation is now similar to that of Lemma A.3.

Case 2. $n>n_{j-p+1}$. In this case, assume that for some $B=B^{c}$ we have

$$
\left|v_{j}^{2}-v_{j-p}^{2}\right|<2^{-n_{j-p+1}+1} \text {. }
$$

Notice that this eventuality always occurs. We have with $l=n-n_{j-p+1}$,

$$
\begin{aligned}
v_{j}^{2}-v_{j-p}^{2}= & \frac{B-2^{l} B_{j-p+1}}{2^{n} \delta}+\frac{v_{j-p+1}}{2^{n_{j-p+1}-1}}-\frac{v_{j+1}}{2^{n-1}} \\
= & \left(B-B^{c}\right) 2^{-n} \delta^{-1}+\left(B^{c}-2^{l} B_{j-p+1}\right) 2^{-n} \delta^{-1} \\
& +v_{j-p+1} 2^{1-n_{j-p+1}}-v_{j+1} 2^{1-n} \\
= & \left(B-B^{c}\right) 2^{-n} \delta^{-1}+\mathcal{O}\left(2^{-n_{j-p+1}}\right) .
\end{aligned}
$$

We now separate the summation on $B$ into two parts. The first one is over those $B$ such that $\left|B-B^{c}\right|>2^{l} \delta$, the second one over those $B$ such that $\left|B-B^{c}\right| \leqq 2^{l} \delta$. In the case of the first sum, we obtain

$$
\begin{aligned}
& \sum_{\substack{B, n \in X_{q} X^{2} \\
\left|B-B^{c}\right|>2^{l} \delta}} 2^{-n}\left|v_{j}^{2}-v_{j-p}^{2}\right|^{-1 / 2} \\
& \quad \leqq \sum_{\substack{B, n \in X_{q} l \\
\left|B-B^{c}\right|>2^{l} \delta}} 2^{-n}\left[\left|B-B^{c}\right| 2^{-n} \delta^{-1}\right]^{-1 / 2} .
\end{aligned}
$$

Define $\alpha=1, u=\delta^{-1}$ and $\varrho=2^{-(n-l) / 2}$. The summation is now similar to that of Lemma A.3. Notice also that if $2^{l} \delta<1$, the second sum is zero. If $2^{l} \delta \geqq 1$, we have for the second sum from Lemma 2.6,

$$
Y \equiv \sum_{\substack{B, n \in X_{q} \\\left|B-B^{c}\right| \leqq 2^{2} \delta}} 2^{-n}\left|v_{j}^{2}-v_{j-p}^{2}\right|^{-1 / 2} \leqq \vartheta 2^{-n} 2^{n_{j-p+1} / 2} \Delta^{8(j-p+1)},
$$

where $\vartheta=\operatorname{Card}\left\{B \mid B\right.$ is $(n, \delta)$-compatible, and $\left.\left|B-B_{c}\right| \leqq 2^{l} \delta, \delta \in \mathbf{K}_{q}\right\}$. From Lemma A. 2 and $2^{l} \delta>1$, we obtain

$$
\begin{aligned}
Y & \leqq 2^{l} \mathcal{O}(1) 2^{-\delta(l-\Delta)} \delta 2^{-n} 2^{n_{j-p+1} / 2} \Delta^{8(j-p+1)} \\
& \leqq \delta \mathcal{O}(1) 2^{-n_{j-p+1} / 2} 2^{-\delta(l-\Delta)} \Delta^{8(j-p+1)} \\
& \leqq \delta^{3 / 2} \mathcal{O}(1) 2^{-\delta l} \Delta^{8(j-p+1)} .
\end{aligned}
$$

Summing over $l$, this is bounded by $\mathcal{O}(1) \delta^{1 / 2} \Delta^{8(j-p+1)}$, and this ends the proof of the lemma. 
Acknowledgements. This work was done at IHES, Rockefeller University, Harvard University and University of Geneva, and financial support was received from NSF Grant PHY-77-18762 and from the Fonds National Suisse. We thank all these institutions for their support, and our colleagues for hospitality and encouragement. An earlier version of this paper contained a proof of existence of an absolutely continuous invariant measure for $f_{\delta}$. We thank J. Guckenheimer for pointing out an error in that proof.

\section{References}

1. Shaw, R.: Strange attractors, chaotic behavior, and information flow (preprint)

2. Lorenz, E.N.: On the prevalence of aperiodicity in simple systems (preprint)

3. Guckenheimer, J.: Commun. Math. Phys. 70, 133-160 (1979)

4. Singer, D. : SIAM J. Appl. Math. 35, 260 (1978)

Communicated by D. Ruelle

Received August 25, 1979; in revised form October 20, 1979 